\title{
Numerical Modeling of Partial Discharges in a Solid Dielectric-bounded Cavity: a Review
}

\author{
Cheng Pan ${ }^{1,2}$, George Chen ${ }^{2}$, Ju Tang ${ }^{1}$ and Kai Wu ${ }^{3}$ \\ ${ }^{1}$ School of Electrical Engineering, Wuhan University, Wuhan 430072, China \\ ${ }^{2}$ School of Electronics and Computer Science, University of Southampton, Southampton SO17 1BJ, UK \\ ${ }^{3}$ State Key Laboratory of Electrical Insulation and Power Equipment, \\ Xi'an Jiaotong University, Xi'an 710049, China
}

\begin{abstract}
Partial discharge (PD) taking place in a solid dielectric-bounded cavity involves physical processes such as free electron supply, discharge development and surface charge decaying, which bring about memory effects and become the main reasons for stochastic behavior of PDs. This paper reviews numerical modeling of cavity PD in the past 30 years. In the first place, physical processes relevant to PD activity are summarized, and modeling methods for discharge development are classified. Then some differences of PD modeling at $\mathrm{AC}$ and DC voltages are distinguished. Subsequently, reproducing methods from simulations to experiments are introduced, as well as their comparison under different conditions and with the emphasis on voltage frequency and PD aging. At last, some problems about current simulation models are discussed, and our suggestions for future work are proposed.
\end{abstract}

Index Terms - partial discharge (PD), cavity PD, streamer, surface charge, free electron

\section{LIST OF MAIN SYMBOLS}

Note: the number in brackets represents the equation numbering where a symbol first appears.

$\alpha$, the gas ionization coefficient

$\beta$, the recombination coefficient (43)

$\beta_{0}$, the exponent in approximation for effective ionization coefficient (7)

$\gamma_{\mathrm{s}}$, the surface conductivity (26)

$\gamma_{\text {gas }}$, the gas conductivity (40)

$\gamma_{\text {hgas }}$, the conductivity of discharge channel

$\gamma_{\text {shigh, }}$, the surface conductivity when the total amount of charges exceed a critical level

$\gamma_{\text {slow }}$, the surface conductivity when the total amount of charges are below a critical level

$\gamma_{\mathrm{pol}}$, the bulk conductivity of insulator

$\varepsilon_{0}$, the vacuum permittivity (8)

$\varepsilon_{\mathrm{r}}$, the relative permittivity

$\varepsilon_{\mathrm{pol}}$, the permittivity of insulator

$\xi$, the fraction of charge detrapping from surfaces (10)

$\xi_{+}, \xi_{-}$, the fraction of charge detrapping respectively from positive and negative surfaces

$\eta$, the gas attachment coefficient

$\lambda$, a dimensionless function to calculate the induced charge (33)

$\rho_{0}$, the air density relative to its value at $p=1.013 \times 10^{5} \mathrm{~Pa}$ and $T=293 \mathrm{~K}(2)$

$\rho_{\mathrm{v}}$, the volume charge density (33)

Manuscript received on 26 November 2018, in final form 8 January 2019, accepted xx Month 2019. Corresponding author: George Chen. $\rho_{\text {gas }}$, the mass density of gas (6)

$\sigma_{\mathrm{s}}$, the surface charge density (33)

$\tau_{\text {lag, }}$, the discharge time lag (4)

$\tau_{\mathrm{dt}}$, the decay time constant for detrappable charges (10)

$\tau_{\mathrm{dc}}$, the time constant for charging the cavity (48)

$\tau_{\mathrm{vol}}$, the time constant for surface charge decay through insulator bulk

$\varphi$, the phase angle

$\varphi_{\mathrm{s}}$, the electric potential on cavity surface (57)

$\Phi$, the effective work function (8)

$C_{\mathrm{a}}$, the capacitance of healthy insulation

$C_{\mathrm{b}}$, the capacitances of void-free insulation in series with the cavity

$C_{\mathrm{c}}$, the capacitance of cavity

$C_{\text {tot }}$, the total capacitance between electrodes (30)

$\boldsymbol{D}$, the vector of electric displacement field (39)

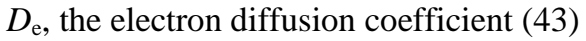

$d_{\text {cav }}$, the cavity height parallel to the applied field (1)

$e$, the elementary charge (8)

$\boldsymbol{E}_{\mathbf{t}}$, the vector of tangential electric field on the cavity surface (58)

$E_{\text {cav }}$, the electric field within the cavity (4)

$E_{\text {cbef }}$, the field within the cavity prior to the PD (36)

$E_{\text {ext }}$, the extinction field (23)

$E_{\text {app }}$, the applied filed

$E_{\text {scha, }}$, the electric field induced by surface charges

$E_{\text {inc }}$, the inception field (1)

$(E / p)_{\mathrm{cr}}$, the pressure reduced critical field at which $\alpha=\eta$ (1)

$\boldsymbol{J}$, the vector of conduction current density (38)

$k_{\mathrm{b}}$, the Boltzmann constant (8)

$N_{\mathrm{e}}$, the electron number density (41) 
$N_{\mathrm{p}}$, the positive ion number density (44)

$N_{\mathrm{n}}$, the negative ion number density (45)

$N_{\mathrm{dt}}$, the number of detrappable charges (9)

$N_{\mathrm{e} 0}$, a constant (17)

$N_{\mathrm{HW}}$, the average discharge number per ac half cycle

$N_{\mathrm{AW}}$, the average discharge number per cycle

$\Lambda_{\mathrm{e}}^{\&}$, the total free electron generation rate (11)

$N_{\mathrm{ev}}^{\&}$, the free electron generation rate by gas radiative

ionization (5)

$N_{\mathrm{es}}^{\&}$, the free electron generation rate by surface detrapping (8)

$p$, the gas pressure within the cavity (1)

$P_{\mathrm{d}}$, the discharge probability (4)

$q_{\mathrm{s}}$, the total charges on the cavity surface (26)

$q_{\text {sfree }}$, the free surface charges (27)

$q_{\text {phy }}$, the physical charge (29)

$q_{\text {app }}$, the apparent charge (30)

$q_{\text {ind }}$, the induced charges at an electrode (33)

$q_{\text {phy }}^{\text {pre }}$, the physical charges from previous PD (10)

$q_{\text {app }}^{\min }$, the minimum apparent charge

$q_{\text {app }}^{\max }$, the maximum apparent charge (42)

$r_{\text {cav }}$, the radius of spherical cavity (7)

$R_{\mathrm{a}}$, the resistance of healthy insulation

$R_{\mathrm{b}}$, the resistance of solid dielectrics in series below and above the cavity

$R_{\mathrm{c}}$, the resistance through and around the cavity

$S_{\text {cav }}$, the area of cavity surface (8)

$S_{\text {det }}$, the function of surface emission law (8)

$S_{\mathrm{sec}}$, the source term about secondary process (43)

$S_{\mathrm{ph}}$, the photoionization source term (46)

$T$, the temperature (3)

$t$, the time

$t_{\mathrm{PD}}$, the moment when a PD starts

$\triangle t_{\text {inc }}$, the time past after the field within the cavity exceeding the inception value (4)

$\triangle t_{\text {pre }}$, the time past after the previous discharge (10)

$\triangle t_{\mathrm{PD}}$, the time duration of PD

$U_{\text {cav }}$, the voltage across the cavity

$U_{\text {ext }}$, the extinction voltage of cavity

$U_{\text {app }}$, the applied voltage across electrodes

$U_{\text {inc }}$, the cavity voltage when the inception field is reached (2)

$U_{\text {cbef, }}$, the voltage across the cavity before a PD

$U_{\text {caft }}$, the voltage across the cavity after a PD

$\triangle U_{\mathrm{c}}$, the difference of voltage across the cavity instantly before and after a PD (29)

$\triangle U$, the difference of voltage across electrodes instantly before and after a PD (30)

$V_{\text {eff, }}$, the effective ionization volume (5)

$V_{\text {cav }}$, the cavity volume (33)

$\boldsymbol{W}_{\mathrm{e}}$, the vector of electron drift velocity (43)

$W_{\mathrm{p}}$, the vector of positive ion drift velocity (44)

$\boldsymbol{W}_{\mathrm{n}}$, the vector of negative ion drift velocity (45)

\section{INTRODUCTION}

PARTIAL discharges (PDs) taking place in a cavity surrounded by solid dielectrics, named cavity PDs, void PDs or internal PDs, have attracted much attention mainly due to two reasons. Firstly, the ion bombardment, localized temperature rise and excited molecules induced by PDs will deteriorate the insulating performance of solid dielectrics, and finally lead to the insulation failure [1, 2]. Secondly, PD behaviors are closely relevant to the characters of solid dielectrics, so the PD measurement is frequently used to diagnose the insulation status of power equipment [3]. To better understand the hazards of PD or make full use of it, PD mechanisms must be clarified at first.

Since the three-capacitor model was proposed, numerical modeling has been widely used to pursue PD mechanisms. Compared with experiments, it has several merits: (i) to obtain some microscopic physical processes easily, whereas they are difficult or even impossible for experimental methods, e.g. the streamer propagation within a cavity; (ii) to adjust any parameter freely so that the critical parameters and physical processes influencing PD behaviors can be identified; (iii) to comprehensively construct relationships of cavity parameters, solid dielectric characteristics, aging status and test conditions with PD events.

Up to now, a large number of models for cavity PD have been proposed. To the best of the authors' knowledge, the earliest numerical modeling of cavity PDs dates back to 1980s [4]. The pioneers' work was based on the point of view of circuit, by which the gas breakdown was reduced to charging-discharging of capacitors. L. Niemeyer proposed a representative approach to model PD in 1990s [5], involving not only the gas breakdown but also the physical processes before and after a discharge, e.g. the supply of free electrons, surface charge decay between two adjacent discharges. Because of the development of finite element method (FEM), some models for the gas breakdown are proposed instead of Niemeyer's analytic model, which are mainly categorized as conductance [6, 7] and electrostatic models [8]. As for the former, the gas breakdown is represented by an increase of gas conductivity, whereas it is considered as the deployment of charges on cavity surface for the latter. Some modeling methods initially designed for dielectric barrier discharge (DBD) are also applied to PD simulation after the 2000s $[9,10]$ owing to the similarity between cavity PD and filamentary DBD.

PD numerical modeling is a traditional topic, but many people are still trying their efforts to develop it now [11-14]. We try to summarize relevant researches in the past 30 years so as to inspire forthcoming studies. First of all, in order to avoid misunderstanding, the scope is restricted. If no otherwise specified, the cavity PD refers to the "streamer" or "streamerlike" discharge in the paper, because it normally induces signals on a level which is detectable in power equipment, and it is usually considered to pose a much more threat to the insulation. The "streamer" or "streamer-like" discharge is characterized as a larger apparent charge $(>10 \mathrm{pC})$ and a shorter pulse duration (1 100 ns) [5], which differs from the Townsend discharge and swarming partial microdischarge (SPMD). In addition, PDs occurring in a narrow channel, e.g. electrical tree, are excluded. For this type of $\mathrm{PD}$, the discharge development is easily affected by channel walls and is difficult to reach channel ends, so its simulation methods are different from the cavity PD [1517].

The paper is organized in a logic order of PD numerical modeling. In the second part, the physical processes before and 
after gas breakdown are introduced, which include the free electron supply, the derivation of PD inception and extinction fields, and surface charge decay between two adjacent discharges. In the third part, various simulation models for discharge development are classified, such as capacitance, electrostatic, conductance and plasma models, merits and drawbacks of which are also discussed. The differences of PD modeling method at AC and DC voltages are distinguished in the fourth part. In the fifth part, methods about how to reproduce experiments from simulations are introduced, and their comparison under different test conditions is presented, with the emphasis on voltage frequency and aging status. In the sixth part, some problems about combination of physical processes, parameters setting, stochastic nature and memory effects are discussed. At last, the review is briefly summarized, and some suggestions for future work are put forward.

\section{PHYSICAL PROCESSES BEFORE AND AFTER THE DISCHARGE DEVELOPMENT}

It is generally known that two conditions must be met to ensure the occurrence of gas breakdown: electric field exceeding a critical value, and free electron supply, so they should be confirmed at first. Besides, the condition for discharge extinguishment needs to be explicitly set in many cases. As usual, the electric field within the cavity is compared with a critical value named extinction field [18]. If the field falls below the extinction one, it is assumed that the gas breakdown is terminated. Hence, the derivation of extinction field is also crucial. After a discharge, there are a large number of charges distributed in the cavity, which will affect the characteristics of subsequent PDs by changing the electric field distribution and additionally providing free electrons for them. The majority of these charges will land on the cavity surface in less than $0.1 \mu \mathrm{s}$ [19], much shorter than the time interval between two adjacent discharges (generally exceeds $0.1 \mathrm{~ms}$ ). Therefore, the surface charge dynamics should be highlighted in PDs analysis.

\subsection{PD INCEPTION FIELD}

On one hand, considering the streamer type of PD in essence, the inception field $E_{\text {inc }}$, defined as the minimum electric field required for a $\mathrm{PD}$ to take place, is derived from the critical avalanche criterion [5, 20, 21], and has the following expression:

$$
E_{\text {inc }}=(E / p)_{\text {cr }} p\left[1+\frac{B}{\left(p d_{\text {cav }}\right)^{n}}\right]
$$

where $(E / p)_{\mathrm{cr}}$ is the pressure reduced critical field at which $\alpha=\eta$, $\alpha$ is the gas ionization coefficient, $\eta$ is the gas attachment coefficient, $p$ indicates the gas pressure within the cavity, $B, n$ are constants, and $d_{\text {cav }}$ indicates the cavity height parallel to the applied field. If the cavity is full of air, $(E / p)_{\mathrm{cr}}=25.2 \mathrm{VPa}^{-1} \mathrm{~m}^{-1}$, $B=8.6 \mathrm{~m}^{0.5} \mathrm{~Pa}^{0.5}$ and $n=0.5$. Additionally, the inception voltage $U_{\text {inc }}$ is also considered as the breakdown value across two electrodes with a uniform electric field distribution by some researchers [22, 23]. The expression is obtained by experimental data fitting [24], in accordance with Paschen's law.

$U_{\text {inc }}=24.41\left(\rho_{0} d_{\text {cav }}\right)+6.73 \sqrt{\rho_{0} d_{\text {cav }}}$ and $\rho_{0}=\frac{p}{1.013 \times 10^{5}} \cdot \frac{293}{T}$

where $\rho_{0}$ indicates the air density relative to its value at $p=1.013$ $\times 10^{5} \mathrm{~Pa}$ and $T=293 \mathrm{~K}, U_{\text {inc }}$, in $\mathrm{kV}$, indicates the cavity voltage when the inception field is reached and $d_{\text {cav }}$ is in $\mathrm{cm}$. Figure 1 shows the change of inception field with cavity height. The results obtained by equation (1) are slightly higher than by Equation (2). Note that Paschen's law means Townsend type discharge in essence, which is not in accordance with the streamer type's assumption.

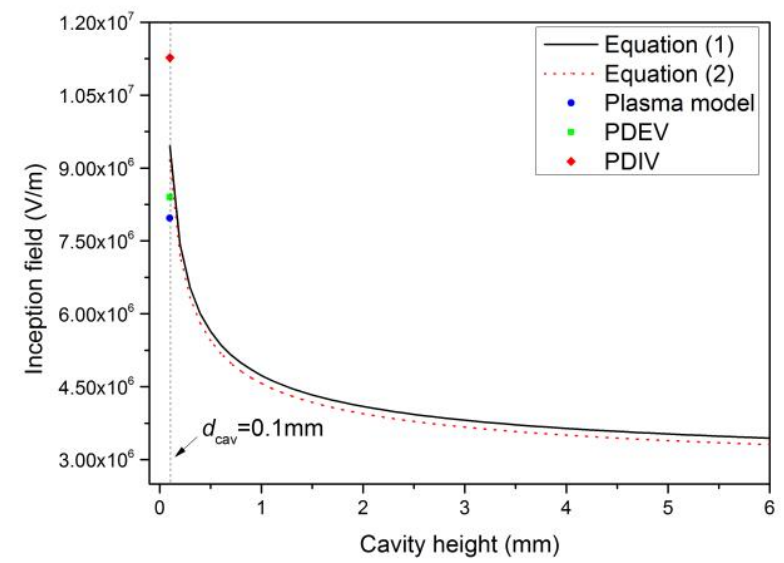

Figure 1. Change of inception field with cavity height. $p=1.013 \times 10^{5} \mathrm{~Pa}$, $T=293 \mathrm{~K}$. The measurement results are from [26].

On the other hand, the inception voltage can be determined based on PD measurement. Some people thought it approximately equals to the partial discharge inception voltage (PDIV) $[8,25]$, while some argued that it should be determined by the partial discharge extinction voltage (PDEV) [26]. In terms of plasma models mentioned later, G. Callender et al obtained the lowest voltage where free electrons could be developed into a PD within a cylindrical cavity of $0.1 \mathrm{~mm}$ in height [26]. Its value of $1.49 \mathrm{kV}$, corresponding to a mean field of $7.97 \mathrm{kV} / \mathrm{mm}$ in the cavity, approximately equals to the value of PDEV, but is obviously lower than the PDIV and the voltage obtained by Equation (1), as shown in Figure 1. The higher value of PDIV may be attributed to the effect of discharge time lag [27].

\subsection{FREE ELECTRON SUPPLY}

Due to the requirement of free electrons to start the avalanche of ionization process, there is usually a time delay between the instant of application of an electric field in excess of the static breakdown field and the onset of gas breakdown. Strictly speaking, the time delay comprises of two parts: the statistical time lag and the formative time lag [24]. The latter involves the time interval from the first electron multiplication to the ultimate breakdown, while the former contains the time spent on waiting until a free electron appears in the cavity and the time it takes to initiate an avalanche process [28]. Compared with the first part of statistical time lag (in the level of ms or longer), the second part and the formative time lag (in the level of ns for a cavity discharge) could be neglected under a usual AC voltage with the frequency less than $\sim 100 \mathrm{kHz}$. Therefore, the discharge time lag roughly refers to the first part of statistical time lag, which depends on the free electron supply. 
Initially, the discharge time lag was explicitly considered for PD simulations due to its simplicity and the uncertainty of free electron generation [4, 29]. It was usually assumed that there is an exponential relationship between discharge time lag $\tau_{\text {lag }}$ and discharge probability $P_{\mathrm{d}}$, as follows [30]:

$$
P_{\mathrm{d}}=\left\{\begin{array}{cc}
1-\exp \left(-\frac{\Delta t_{\text {inc }}}{\tau_{\text {lag }}}\right) & E_{\text {cav }} \geq E_{\text {inc }} \\
0 & E_{\text {cav }}<E_{\text {inc }}
\end{array}\right.
$$

where $\triangle t_{\text {inc }}$ indicates the time past after the field within the cavity exceeding the inception value, and $E_{\text {cav }}$ is the electric field within the cavity. In the case of $\tau_{\text {lag }}$ and other parameters remaining known, $P_{\mathrm{d}}$ is calculated, and then compared with a random number $x$ between 0 and 1 . If $P_{\mathrm{d}}$ exceeds this number, the conditions for discharge occurrence are satisfied.

Obtaining the characteristics of discharge time lag becomes a precondition for $P_{\mathrm{d}}$ calculation. Although it had been proved to be an inverse power dependence on the applied voltage by experiments and simulations [31, 32], some doubts should be specified. Firstly, the experiments were conducted under step voltage, and only the discharge time lag for the first PD was measured [31]. Without considering memory effects, the conclusion may not apply to continuous PDs. Secondly, the simulation results were obtained from a simplified model, which needs to be validated [32].

Discharge time lag is actually the inverse of the production rate of free electrons. Since the generation mechanism was clarified, most researchers have used it for PD simulation. In general, two ways of free electron supply are distinguished, the volume generation and the surface emission [5]. The former consists of gas radiative ionization and field detachment of electron from negative ions. In a cavity surrounded by solid dielectrics, the gas radiative ionization is dominant, and the free electron generation rate is expressed as:

$$
\begin{aligned}
& N_{\mathrm{ev}}^{\&}(t)=\eta_{\text {irad }} p V_{\text {eff }}\left(1-\frac{\eta}{\alpha}\right) \\
& \eta_{\text {irad }}=C_{\text {rad }} \psi_{\text {rad }} \rho_{\text {gas }} / p
\end{aligned}
$$

where $\eta_{\text {irad }}$ indicates the radiative ionization, $V_{\text {eff }}$ is the effective ionization volume, and the last term, named Legler function, indicates the probability that an electron develops into an avalanche. In the case of local field exceeding the inception field, $\alpha \gg \eta$, so the Legler function approximately equals to unity. $C_{\text {rad }}$ shows the interaction between radiation and gas, $\psi_{\mathrm{rad}}$ is the radiation quantum density, and $\rho_{\text {gas }}$ indicates the mass density of gas.

For a spherical cavity, the effective ionization volume has the following expression,

$V_{\text {eff }}=\frac{4}{3} \pi r_{\text {cav }}^{3}\left(1-\kappa^{-\beta_{0}}\right)$

where $r_{\text {cav }}$ indicates the radius of spherical cavity, $\beta_{0}$ is the exponent in approximation for effective ionization coefficient, and $\kappa=U_{\text {cav }} / U_{\text {inc. }} U_{\text {cav }}$ is the voltage across the cavity.

Free electrons emit from the cavity surface by detrapping, ion impact release and surface photo effect. Among them, the first source is usually dominant, which obeys the RichardsonSchottky scaling,

$$
\begin{aligned}
& S_{\mathrm{es}}^{\&}(t)=\frac{S_{\mathrm{cav}}}{e} S_{\mathrm{det}} \exp \left(-\frac{\Phi-\sqrt{e^{3} E /\left(4 \pi \varepsilon_{0}\right)}}{k_{\mathrm{b}} T}\right)\left(1-\frac{\eta}{\alpha}\right) \\
& S_{\mathrm{det}}=v_{0} e \frac{N_{\mathrm{dt}}}{S_{\mathrm{cav}}}
\end{aligned}
$$

where $S_{\text {cav }}$ indicates the area of cavity surface, $e$ is the elementary charge, $S_{\text {det }}$ is the function in surface emission law, $\Phi$ is the effective work function, $\varepsilon_{0}$ is the vacuum permittivity, $k_{\mathrm{b}}$ is the Boltzmann constant, $v_{0}$ is the fundamental phonon frequency, and $N_{\mathrm{dt}} / S_{\mathrm{cav}}$ represents the surface density of detrappable charges. $N_{\mathrm{dt}}$ is assumed to be proportional to the charges generated by previous PD. Due to the loss mechanism, e.g. diffusion into deep traps, the charges will decay until subsequent PD takes place. In general, an exponential function is used to characterize the loss as:

$N_{\mathrm{dt}}=\xi\left(q_{\text {phy }}^{\text {pre }} / e\right) \exp \left(-\Delta t_{\text {pre }} / \tau_{\mathrm{dt}}\right)$

where $\xi$ is the fraction of $q_{\text {phy }}^{\text {pre }}$ in a detrappable state, $q_{\text {phy }}^{\text {pre }}$ indicates the physical charges from previous $\mathrm{PD}, \Delta t_{\mathrm{pre}}$ is the time past after the previous discharge, and $\tau_{\mathrm{dt}}$ indicates the decay time constant. Therefore, the total rate of free electron generation is:

$N_{\mathrm{e}}^{\&}=N_{\mathrm{ev}}^{\&}+N_{\mathrm{es}}^{\&}$

In the case of spherical or ellipsoidal cavity, free electrons for the first PD mainly come from the volume generation, while the primary source is surface emission for a flat cavity due to its large surface-to-volume ratio [33]. After the first PD has occurred, the surface emission becomes dominant. We compare the free electron production rate from the two ways, as shown in Figure 2. It is found the surface generation rate is much higher than the volume even if $\Delta t_{\mathrm{pre}} / \tau_{\mathrm{dt}}<15$, so the surface detrapping dominates free electron supply in most cases.

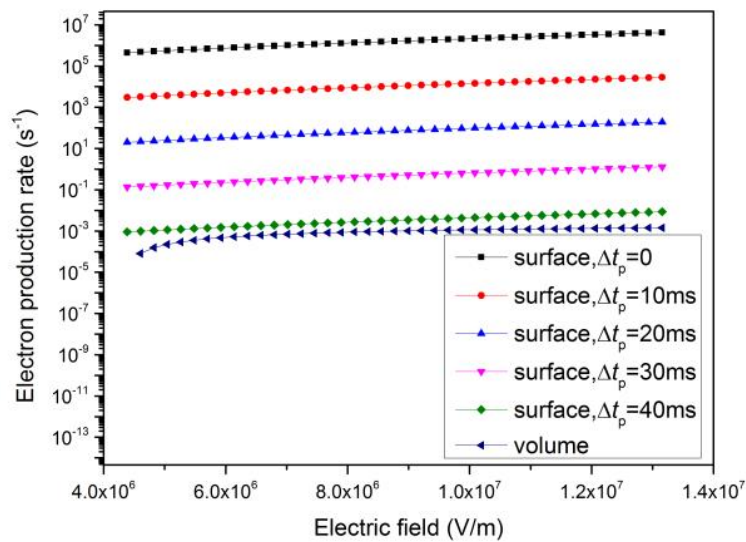

Figure 2. Change of electron production rate with cavity field. $C_{\mathrm{rad}} \psi_{\mathrm{rad}}=2 \times 10^{6}$ $\mathrm{kg}^{-1} \mathrm{~s}^{-1}, \rho_{\text {gas }}=1.18 \mathrm{~kg} / \mathrm{m}^{3}, p=1.01 \times 10^{5} \mathrm{~Pa}, r_{\text {cav }}=0.7 \mathrm{~mm}, \xi=0.02, q_{\text {phy }}^{\text {pre }}=100 \mathrm{pC}$, $e=1.6 \times 10^{-19} \mathrm{C}, \tau_{\mathrm{dt}}=2 \mathrm{~ms}, v_{0}=10^{14} \mathrm{~s}^{-1}, \Phi=1 \mathrm{eV}, \varepsilon_{0}=8.85 \times 10^{-12} \mathrm{~F} / \mathrm{m}, k_{\mathrm{b}}=1.38 \times 10^{-23}$ $\mathrm{J} / \mathrm{K}, T=300 \mathrm{~K}$, and $E_{\text {inc }} \leq E_{\text {cav }} \leq 3^{*} E_{\text {inc. }} E_{\text {inc }}$ is obtained by equation (1), and the values of most parameters are from [5].

The Richardson-Schottky expression has been adopted in PD simulation, but it should be noted that the expression is based on the earlier measurement of electron emission from concentric glass tubes in the vacuum [5]. Whether or not it is valid for trapped charges on cavity surface needs to be verified. Equations (5)-(10) are usually modified for PD simulation. Table 1 summarizes these modifications from different 
researchers chronologically, as well as how to calculate the

from various research groups, which are usually determined by discharge probability. The expressions of $P_{\mathrm{d}}$ differ significantly

the comparison of simulations with experimental results.

Table 1. The formulas for free electron supply and discharge probability.

\begin{tabular}{|c|c|c|c|}
\hline Ref. & Volume generation & Surface emission & Discharge probability \\
\hline [5] & Equation (5) & Equation (8) & $P_{\mathrm{d}}(t)=1-\exp \left(-N_{\mathrm{e}}^{\&} \Delta t_{\mathrm{inc}}\right)$ \\
\hline [34] & $x$ & Equation (8) & $P_{\mathrm{d}}(t)=1-\exp \left\{-\left[\frac{\alpha_{\mathrm{es}}^{\&} \Delta t_{\mathrm{inc}}}{\alpha_{1}}\right]^{\beta_{1}}\right\}(13)^{\mathrm{a}}$ \\
\hline [35] & Equation (5) & Equation (8) & $P_{\mathrm{d}}(t)=\sum_{i=1}^{m} p_{i}\left\{1-\exp \left[-\left(\frac{\alpha_{\mathrm{e}}^{\&} \Delta t_{\text {inc }}}{\alpha_{i}}\right)^{\beta_{i}}\right]\right\}(14)^{\mathrm{b}}$ \\
\hline [6] & $x$ & $N_{\mathrm{es}}^{\&}(t)=N_{\mathrm{e} 0} \exp \left|U_{\mathrm{cav}}(t) / U_{\mathrm{inc}}\right|(17)^{\mathrm{d}}$ & $P_{\mathrm{d}}(t)=1-\exp \left(-\int_{0}^{t} N_{\mathrm{es}}^{\&}\left(t^{\prime}\right) \mathrm{d} t^{\prime}\right)$ \\
\hline [7] & $\times$ & $\begin{array}{c}N_{\mathrm{es}}^{\&}(t)=\left[N_{\mathrm{ed}}^{\&}(t)+N_{\mathrm{ei}}^{\&}\right] \exp \left|U_{\mathrm{cav}}(t) / U_{\mathrm{inc}}\right|(19)^{\mathrm{e}} \\
N_{\mathrm{ed}}^{\&}(t)=N_{\mathrm{ed} 0}\left|U_{\mathrm{cav}}\left(t_{\mathrm{pre}}\right) / U_{\mathrm{inc}}\right| \exp \left[-\left(t-t_{\mathrm{pre}}\right) / \tau_{\mathrm{dt}}\right](20)^{\mathrm{f}}\end{array}$ & $P_{\mathrm{d}}(t)=N_{\mathrm{es}}^{\&} \Delta t_{\mathrm{inc}}(21)$ \\
\hline [37] & $x$ & Equation (8) & $P_{\mathrm{d}}(t)=1-\exp \left\{-\left[\frac{\ln \left(\left(_{\mathrm{es}}^{\mathrm{ppu}}\right) \Delta t_{\mathrm{inc}}^{\mathrm{pu}}\right.}{\alpha}\right]^{\beta}\right\}(22)^{\mathrm{g}}$ \\
\hline
\end{tabular}

${ }^{\mathrm{a}} \alpha_{1}, \beta_{1}$ are the Weibull parameters of experimental data.

${ }^{\mathrm{b}} m$ is the number of elementary Weibull functions fitting experimental data, and $\alpha_{\mathrm{i}}, \beta_{\mathrm{i}}$ are their parameters.

${ }^{\mathrm{c}} \Phi_{0}, \triangle \Phi$ are the constants, and $\tau_{\text {work }}$ indicates the time constant about temporal evolution of the effective work function.

${ }^{\mathrm{d}} N_{\mathrm{e} 0}$ is a constant.

${ }^{\mathrm{e}} N_{\mathrm{ed}}$ indicates the electron number detrapping from the shallow traps, and $N_{\mathrm{ei}}$ is the electron number detrapping from polymer loose chain ends.

${ }^{\mathrm{f}} N_{\text {edo }}$ is the initial number of detrappable electrons per unit time.

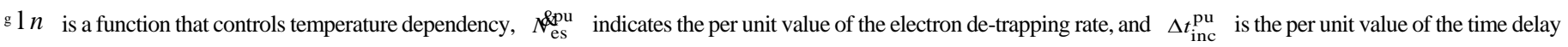
after the voltage exceeds breakdown level.

\subsection{PD EXTINCTION FIELD}

Based on the theory of streamer propagation, the extinction field is estimated as [5]:

$E_{\text {ext }}=\chi E_{\text {cr }}$

where the dimensionless proportionality factor $\chi$ is related to the gas, dielectric surface and streamer polarity. As for the above equation, there are several points to note. Firstly, $\chi=0.2$ and 0.5 respectively for the positive and negative streamers are usually employed in the PD simulations [39, 40], but the two values may not apply to the system made up of gas and polymer. Secondly, $E_{\text {cr }}$ represents the field at which $\alpha=\eta$, not equal to $E_{\text {inc }}$ [25]. In addition, G. Callender et al argued that Equation (23) is derived from a metallic needle-plane system, and may be not applicable for the cavity PD [14]. In terms of a plasma model, they found that the residual field after a PD is around 0.1 $\mathrm{kV} / \mathrm{mm}$, whereas the extinction field predicted by equation (23) is in the order of $1 \mathrm{kV} / \mathrm{mm}$. Moreover, the residual field is not constant, but varies with the electric field instantly before the PD [26].

According to the dependence of PD magnitude on the temperature, F. Haghjoo et al. inferred that $\chi$ is sensitive to the temperature [37]. Therefore, it is developed into

$$
\chi(T)=\chi_{0}-\frac{T}{T_{0}}
$$

where $\chi_{0}$ and $T_{0}$ are constants. Furthermore, Illias thought the extinction field depends on the gas pressure [41],

$E_{\text {ext }}=E_{\text {exto }} p / p_{0}$

where $E_{\text {ext0 }}, p_{0}$ are constants. For a non-vented cavity, the two equations are actually consistent.

The extinction field can be determined from the measured minimum discharge magnitude $q_{\text {app }}^{\min }$, because $q_{\text {app }}^{\min }$ appears at the inception field, and is proportional to the field drop $E_{\mathrm{inc}}-E_{\mathrm{ext}}$ [5]. Based on this method, the predicted $E_{\text {ext }}$ is around $1 \mathrm{kV} / \mathrm{mm}$ [8], in accordance with the result from Equation (23).

\subsection{SURFACE CHARGE DECAY}

The surface charges deployed by PD have a finite lifetime. Three mechanisms have been identified for the surface charge decay: electric conduction along the insulator surface, electric conduction through the insulator volume, and charge neutralization by gas ions [42]. The first is widely considered as the dominant way in the cavity PDs [5, 25, 39]. According to Ohm's law, the surface charge decay is approximately described as [39]:

$\frac{\mathrm{d} q_{\mathrm{s}}}{\mathrm{d} t}=-\frac{\pi}{2} \gamma_{\mathrm{s}} E d_{\mathrm{g}}$

where $q_{\mathrm{s}}$ indicates the total charges on the cavity surface, $\gamma_{\mathrm{s}}$ is the surface conductivity, and $E d_{\text {cav }}$ indicates the potential difference across the cavity.

Several doubts should be pointed out for equation (26). At first, some researchers roughly held that $q_{\mathrm{s}}$ is the physical charges from a PD [39], whereas the other argued that it is only restricted to the free charges, and should not include the trapped charges [8]. The free charges are estimated by the following formula:

$q_{\text {sfree }}=\int_{S_{\text {cav }}}\left(D_{\mathrm{s}}^{2}-D_{\mathrm{s}}^{1}\right) \mathrm{d} S$

where $D_{\mathrm{s}}^{1}$ and $D_{\mathrm{s}}^{2}$ indicate the displacement field at gas and solid dielectric sides of the interface, respectively. In addition, the surface conduction is beneficial to a uniform distribution of surface charges, but does not lead to the decrease of total 
charges [42]. Therefore, the mechanism of surface charge decay is actually confusing in the usage of equation (26).

The movement of surface charges along the spherical cavity surface is analyzed, as shown in Figure 3. After a PD takes places, the deployed surface charges will reduce the applied field, but the direction of total field will not change. In this case, the charges tend to move towards the centers of upper and lower surfaces. If the voltage polarity is reversed, the total field will drive positive and negative charges to move towards each other, and the charge recombination will take place, leading to the decrease of total charges. In order to model the processes, Illias et al put forward two methods. One is based on the charge drift and recombination on the cavity surface [8], and the other is based on the adjustment of surface conductivity [7]. If $E_{\mathrm{cav}}$ has the opposite direction to $E_{\text {scha, }}$, a lower value is used; otherwise a higher surface conductivity is employed.

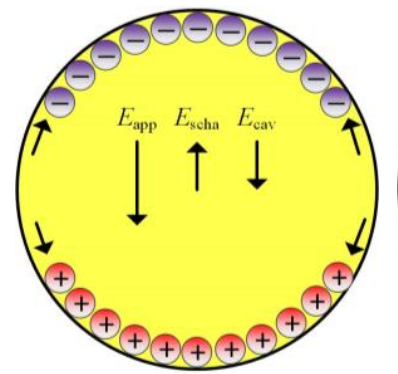

(a) Before the polarity reversal

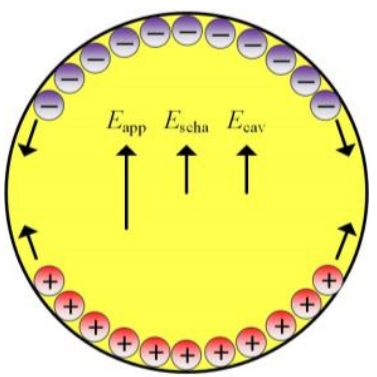

(b) After the polarity reversal
Figure 3. Movement of surface charges due to the surface conductivity. $E_{\text {app }}$ indicates the applied filed, and $E_{\text {scha }}$ is the electric field induced by surface charges. The figure is reproduced from [7].

However, for a flat cavity, the surface charge recombination is difficult to happen due to the separation of cavity walls. In terms of surface conduction, surface charge decay could not be well explained. We constructed a surface charge measurement system based on Pockels effect, and obtained the surface charge dynamic distribution during a PD sequence which took place within a flat cavity [43, 44], as illustrated in Figure 4. It was found that there is no lateral migration of surface charges, and the decay rate of positive charges is much higher than the negative. Due to the complex configuration of electrodes, the surface charge decaying mechanism is not addressed, but it is inferred that the volume conduction and gas ion neutralization contribute to the decay.

The surface charge decay through the insulator volume depends on its bulk conductivity $\gamma_{\mathrm{pol}}$ and permittivity $\varepsilon_{\mathrm{pol}}$, which obeys an exponential law [42, 45],

$q_{\mathrm{s}}=q_{\mathrm{s} 0} \exp \left(-t / \tau_{\mathrm{pol}}\right)$

where $q_{\mathrm{s} 0}$ indicates the initial surface charges, and $\tau_{\mathrm{vol}}=\varepsilon_{\mathrm{pol}} / \gamma_{\mathrm{pol}}$ is the time constant. Take a cavity surrounded by polyethylene (PE) as an example, the relative permittivity $\varepsilon_{\mathrm{r}}=2.3$ and $\gamma_{\mathrm{poo}} \approx 1 \times 10^{-16} \mathrm{~S} \cdot \mathrm{m}^{-1}$ at the room temperature, so $\tau_{\mathrm{pol}} \approx 2 \times 10^{5} \mathrm{~s}$. Compared with the voltage cycle, it is much longer, and the surface charge decay can be neglected.

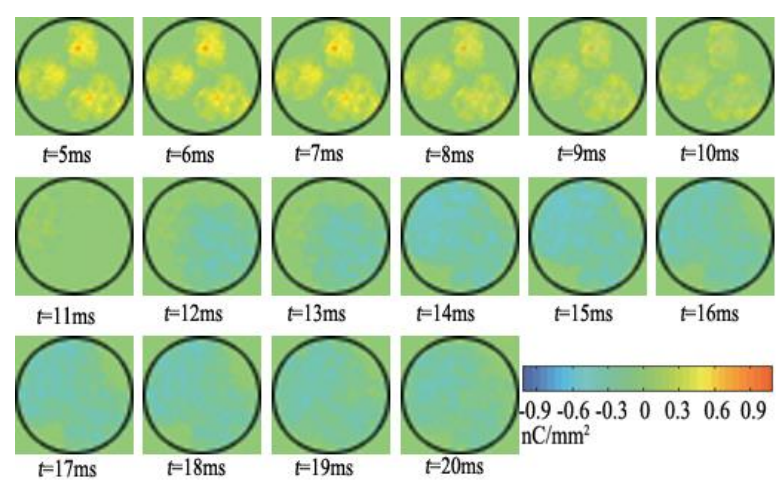

Figure 4. Dynamic distribution of surface charges during a PD sequence with $50 \mathrm{~Hz}$ AC voltage application. The height of cylindrical cavity is $0.15 \mathrm{~mm}$ and the diameter is $1.5 \mathrm{~mm}$. The figure is reproduced from [43].

Due to a very long decay time in the case of natural ionization, e.g. in the order of $1000 \mathrm{~min}$ [42], the surface charge decay caused by gas ion neutralization is usually abandoned. Although the majority charges produced by PD land on the cavity surface, residual space charge density is much higher in comparison with the natural level. After the cavity field reversal, the residual space charges will drift along the opposite direction and neutralize the surface charges, possibly leading to a significant reduction of decay time. S. Kumara et al. investigated the surface charge decay in the case of ion density ten times over the natural level [46]. Compared with the case of natural ionization, the decay rate is 10 times faster during the entire stage for Elastosil, and 2 times faster during the early stage for Powersil.

\section{PHYSICAL MODELS ABOUT DISCHARGE DEVELOPMENT}

\subsection{CAPACITANCE MODELS}

The classical capacitance model, named three-capacitor or ab-c model, was initially proposed by Gemant and Philippoff [47], as shown in Figure 5. The cavity is represented by the capacitance $C_{\mathrm{c}}$. $C_{\mathrm{a}}$, the equivalent parallel capacitance of $C_{\mathrm{a} 1}$ and $C_{\mathrm{a} 2}$, indicates the capacitance of healthy insulation. $C_{\mathrm{b}}$ is a resultant series capacitance of $C_{\mathrm{b} 1}$ and $C_{\mathrm{b} 2}$, which represents the capacitance of void-free insulation in series with the cavity. The occurrence of PD is simulated by turning on the switch $\mathrm{S}$ when the voltage across $C_{\mathrm{c}}$ reaches $U_{\text {inc. }}$. For an improved model developed by Whitehead [48], the switch was replaced by a thyristor controlled device so that the breakdown voltage can be set at any desired value.

In terms of the model, the physical charge $q_{\text {phy }}$ is expressed as:

$q_{\text {phy }}=\Delta U_{\mathrm{c}}\left(C_{\mathrm{c}}+\frac{C_{\mathrm{a}} C_{\mathrm{b}}}{C_{\mathrm{a}}+C_{\mathrm{b}}}\right)$

where $\triangle U_{\mathrm{c}}$ indicates the difference of voltage across the cavity before and after a $\mathrm{PD}$, equal to $U_{\text {cbef- }}-U_{\text {caft. }}$. In addition, the apparent charge $q_{\text {app }}$ is expressed as:

$q_{\text {app }}=\Delta U C_{\text {tot }}$

where $\Delta U$ is the difference of voltage across electrodes before and after a $\mathrm{PD}$, and $C_{\text {tot }}$ is the total capacitance between electrodes. Because $\Delta U$ and $C_{\text {tot }}$ can be measured, the apparent charge is experimentally obtained by the pulse current method. 
Moreover,

$q_{\text {app }}=\Delta U_{\mathrm{c}} \frac{C_{\mathrm{b}}}{C_{\mathrm{a}}+C_{\mathrm{b}}}\left(C_{\mathrm{a}}+\frac{C_{\mathrm{b}} C_{\mathrm{c}}}{C_{\mathrm{b}}+C_{\mathrm{c}}}\right)$

As usual, $C_{\mathrm{a}} \gg C_{\mathrm{c}} \gg C_{\mathrm{b}}$. Therefore,

$$
q_{\mathrm{app}} \approx \Delta U_{\mathrm{c}} C_{\mathrm{b}}
$$

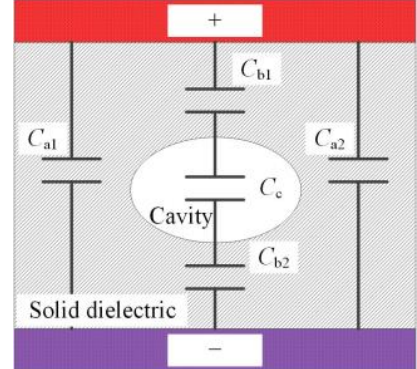

(a) Equivalent circuit
Figure 5. The a-b-c model.

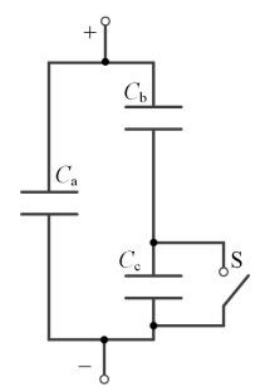

(b) Simplified circuit
Instantly after a $\mathrm{PD}, U_{\text {caft }}$ is equivalent to $U_{\text {ext }}$, so $\Delta U_{\mathrm{c}}=U_{\text {cbef }^{-}}$ $U_{\text {ext }}$, in which $U_{\text {ext }}$ represents the extinction voltage of cavity corresponding to $E_{\text {ext }}$. For a non-constant voltage, $U_{\text {cbef }}$ depends on the discharge time lag. Hence, if the time lag, controlled by the switch, is set, the apparent charge can be calculated. At the initial period of PD simulation, Okamoto et al modeled PD behaviors by such method [4].

Based on the classical a-b-c model, some improved circuits have been proposed. Table 2 summarizes various capacitance models and relevant explanations, in which resistors are sometimes introduced to simulate the conductivity of insulation and discharge channel. When a PD takes place, the cavity resistance will be largely reduced, so a lot of researchers use a time-varying resistor to model the PD development [30, 37, 49]. In addition, circuits including three resistors parallel to capacitors are constructed whereby the effect of charge dissipation on PD can be considered at both $\mathrm{AC}$ and $\mathrm{DC}$ voltages $[38,50]$.

Obviously, the capacitance models are concise, in which the discharge development process is reduced to chargingdischarging of capacitor, so they are easily applied to power equipment, e.g. power cables [49]. However, I. W. McAllister pointed out that the definition of void capacitance is valid only when the cavity surface is equipotential [51]. Due to nonuniform distribution of surface charges, the cavity surface is not equipotential after a PD, which violates the basic definition of capacitance.

Table 2. Various capacitance models for PD simulation.

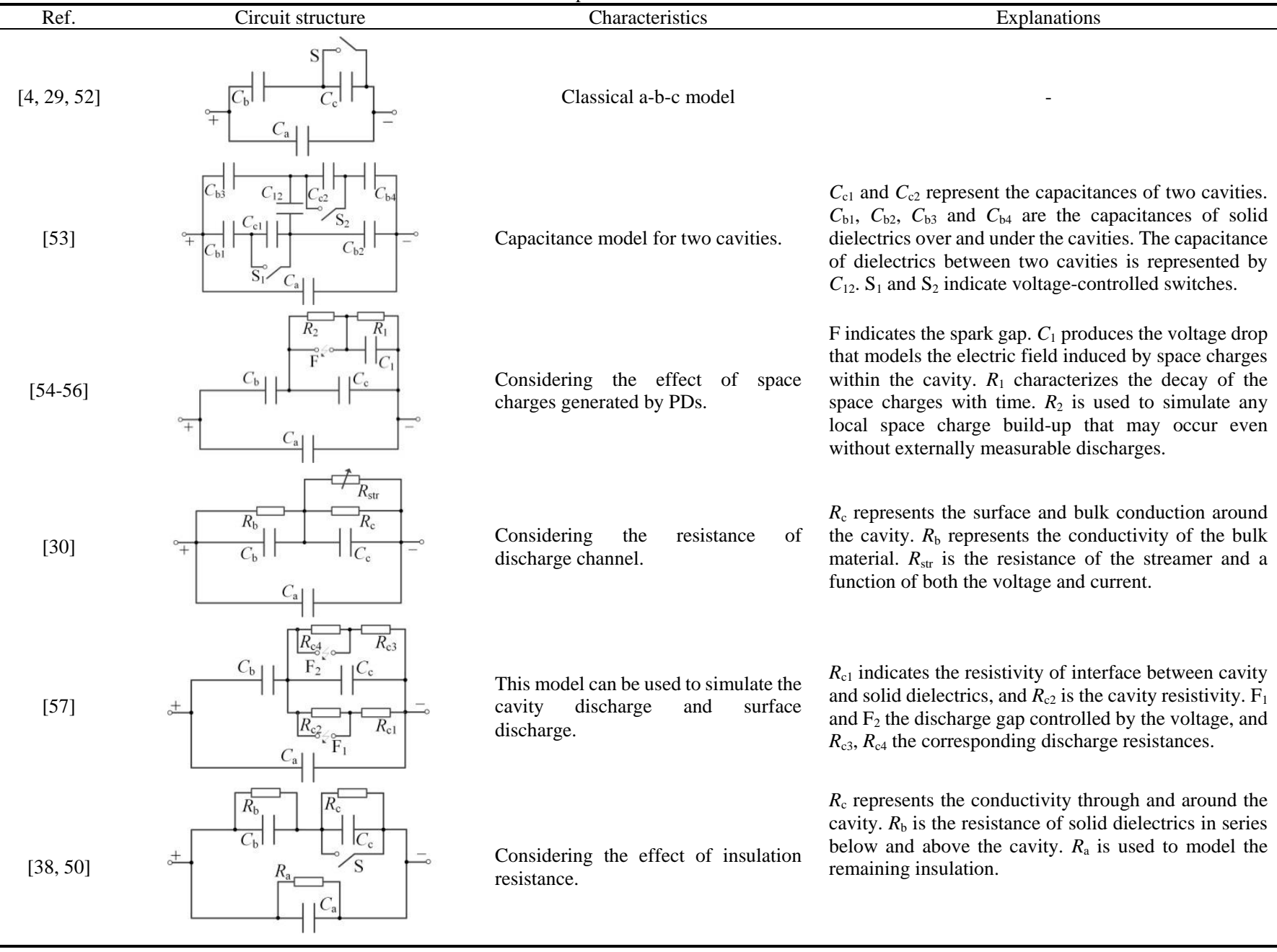




\subsection{ELECTROSTATIC MODELS}

Due to the fallacy of cavity capacitance, the concept of induced charge is introduced to quantify the relationship between physical charge and apparent charge [58, 59]. It is based on the fact that charge transport within the cavity will lead to the variation of surface charges at electrodes during the discharge development. If the charge distribution within the cavity is known at a certain moment, the induced charges can be calculated from

$q_{\text {ind }}(t)=-\iiint_{V_{\text {cav }}} \lambda \rho_{\mathrm{v}}(t) \mathrm{d} V-\iint_{S_{\text {cav }}} \lambda \sigma_{\mathrm{s}}(t) \mathrm{d} S$

where $q_{\text {ind }}$ indicates the entire induced charges at an electrode, $V_{\text {cav }}$ represents the cavity volume, $\rho_{\mathrm{v}}$ and $\sigma_{\mathrm{s}}$ are volume and surface charge density within the cavity, respectively. $\lambda$, a dimensionless function, is expressed as follows:

$\nabla^{2} \lambda=0$

When considering the induced charges at the cathode, $\lambda=1$ at the cathode and $\lambda=0$ at the anode; otherwise $\lambda=0$ at the cathode and $\lambda=1$ at the anode. In addition, at the interface between the cavity and solid dielectrics

$\varepsilon_{1}\left[\frac{\partial \lambda}{\partial e_{n}}\right]_{1}=\varepsilon_{2}\left[\frac{\partial \lambda}{\partial e_{n}}\right]_{2}$

where 1 and 2 represent the gas and solid dielectric, respectively, $e_{n}$ is the normal direction of interface.

If the charge interaction between solid dielectrics and electrodes is neglected, the apparent charge will be equal to the difference of induced charges instantaneously before and after a Niemeyer thought PD leads to the deployment of surface charges, and hetero-charges with the same density are distributed at the upper and lower surfaces [5], as shown in Figure 3. In terms of $\mathrm{PD}$ inception and extinguishment conditions, the surface charge $q_{\mathrm{s}}$ is expressed as:

$q_{\mathrm{s}}=\varepsilon_{0} \pi r_{\text {cav }}^{2}\left(1+2 \varepsilon_{\mathrm{r}}\right)\left(E_{\text {cbef }}-E_{\text {ext }}\right)$

where $E_{\text {cbef }}$ indicates the field within the cavity prior to the PD. From Equations (33)-(36), the apparent charge is obtained [39] as:

$$
q_{\text {app }}=-4 \pi \varepsilon_{0} \varepsilon_{\mathrm{r}} r_{\text {cav }}^{3}\left(E_{\text {cbef }}-E_{\text {ext }}\right) \nabla \lambda
$$

The above derivations is based on the assumption that electric field and surface charge distributions in the cavity are uniform, which is inconsistent with the actual situation. H. A. Illias et al. used an FEM instead of the analytic method to simulate PD [8, $60,61]$. After the inception condition is satisfied, certain charges are added to the cavity surface, and the electric field distribution is calculated by Poisson equation. This process is repeated until the cavity field was below the extinction value.

Considering the expansion of discharge area, Wu et al took the simulation method of electrical tree as reference to construct a PD model [62], in which the streamer is reduced to two points with high charge density on the flat cavity surface at the beginning of PD development [63]. Because of high electric field induced by surface charges, the points would expand along cavity surface, and two critical parameters about electric field are introduced to control the expansion. Based on this model, we refined the streamer propagation, and studied its effect on surface charge accumulation and in turn PD behaviors [23, 64]. In addition, Illias et al also considered the discharge channel expansion along dielectric surface based on the FEM model
[65].

To sum up, the PD development is simplified by the deployment of surface charges, and the Poisson equation (sometimes the analytic formula) is employed to solve electric field distribution in electrostatic models. Compared with the capacitance model, the electrostatic characterizes the PD development from the point of view of field model.

\subsection{CONDUCTANCE MODELS}

During the PD development, there are a large number of charges distributed in the discharge channel. Therefore, the conductivity of cavity is largely increased in comparison with the initial state. In terms of this character, Forssen et al constructed a PD simulation model [6], and then Illias et al developed it [7,32]. In the model, the cavity conductivity is set to be gas conductivity without PD occurrence. After the discharge conditions are satisfied, the conductivity increases to a higher value $\gamma_{\text {hgas. }}$. In this case, the cavity field will decrease, and the discharge development will be terminated in a very short time. The electric field distribution is solved by the combination of current continuity equation and Poisson equation.

$$
\begin{aligned}
& \nabla \cdot \boldsymbol{J}+\frac{\partial \rho_{\mathrm{v}}}{\partial t}=0 \\
& \nabla \cdot \boldsymbol{D}=\rho_{\mathrm{v}}
\end{aligned}
$$

where $\boldsymbol{J}$ indicates the conduction current density, and $\boldsymbol{D}$ is the electric displacement field. Because the electric field distribution partly depends on the cavity conductivity, how to choose $\gamma_{\text {hgas }}$ becomes a vital point for the model. Forssen et al set $\gamma_{\text {hgas }}=10^{-4} \mathrm{~S} / \mathrm{m}$ to avoid too fast changes of electric potential which may bring about numerical calculation problems [6]. In addition, the conductivity is modeled as [66]

$\gamma_{\text {hgas }}=\gamma_{\text {gas }} \exp \left(\left|U_{\text {cav }} / U_{\text {inc }}\right|+\left|I_{\text {cav }} / I_{\text {cri }}\right|\right)$

where $\gamma_{\text {gas }}$ indicates the gas conductivity, $I_{\text {cav }}$ is the conduction current through the discharge channel, and $I_{\text {cri }}$ represents the critical current for avalanche. Illias et al used experimental results to estimate the conductivity [7],

$\gamma_{\text {hgas }}=\mu_{0} e^{2} N_{\mathrm{e}} \lambda_{\mathrm{e}} / m_{\mathrm{e}} c_{\mathrm{e}}$

$N_{\mathrm{e}}=\frac{q_{\text {app }}^{\max }}{4 / 3 e \pi r_{\text {cav }}^{3}}$

where $\mu_{0}$ indicates the coefficient related to electron energy distribution and mean free path, $N_{\mathrm{e}}$ is the electron number density, $\lambda_{\mathrm{e}}$ is the electron mean free path, $c_{\mathrm{e}}$ is the electron thermal velocity, and $q_{\mathrm{app}}^{\max }$ indicates the maximum apparent charge obtained by PD measurement. In Equation (41), the contribution of ions to the electric conductivity can be negligible due to its low drift velocity [67]. However, it is not reasonable that the apparent charge is used to quantify the electron density in the channel.

Similar to capacitance models, the conductance have a wide applicability, e.g. it is easily used to simulate PD for more than one cavity $[68,69]$ and even power equipment [70]. In addition, due to the introduction of electric conductivity, the temperature variation of cavity caused by PDs can be roughly predicted, and its effect on PD behaviors is investigated [41, 71]. 


\subsection{PLASMA MODELS}

Due to the stochastic essence of PD behaviors, PD models are typically interested in obtaining the statistical characteristics for a large number of PDs. Therefore, the complicated discharge development processes are extremely simplified, as has been shown in the previous sections. In order to reveal the PD mechanism, a more rigorous model is needed. Plasma model was initially used to simulate gas discharge across electrodes [72] and dielectric barrier discharge (DBD) [73], and then some researchers employed them to model discharge development for the cavity PD owing to their similarity [14, 74, 75]. In the model, physical processes such as impact ionization, attachment, recombination, diffusion and drift of charges are quantitatively described by fluid equations:

$$
\begin{aligned}
\frac{\partial N_{\mathrm{e}}}{\partial t}= & N_{\mathrm{e}} \alpha\left|\boldsymbol{W}_{\mathrm{e}}\right|-N_{\mathrm{e}} \eta\left|\boldsymbol{W}_{\mathrm{e}}\right|-N_{\mathrm{e}} N_{\mathrm{p}} \beta- \\
& \nabla \cdot\left(N_{\mathrm{e}} \boldsymbol{W}_{\mathrm{e}}-D_{\mathrm{e}} \nabla N_{\mathrm{e}}\right)+S_{\mathrm{sec}} \\
\frac{\partial N_{\mathrm{p}}}{\partial t}= & N_{\mathrm{e}} \alpha\left|\boldsymbol{W}_{\mathrm{e}}\right|-N_{\mathrm{e}} N_{\mathrm{p}} \beta-N_{\mathrm{n}} N_{\mathrm{p}} \beta-\nabla \cdot\left(N_{\mathrm{p}} \boldsymbol{W}_{\mathrm{p}}\right)+S_{\mathrm{sec}} \\
\frac{\partial N_{\mathrm{n}}}{\partial t}= & N_{\mathrm{e}} \eta\left|\boldsymbol{W}_{\mathrm{e}}\right|-N_{\mathrm{n}} N_{\mathrm{p}} \beta-\nabla \cdot\left(N_{\mathrm{n}} \boldsymbol{W}_{\mathrm{n}}\right)
\end{aligned}
$$

where $N$ is the charge number density, e, $\mathrm{p}$ and $\mathrm{n}$ are the symbols for electron, positive ion and negative ion, respectively. $\beta$ and $D_{\mathrm{e}}$ denote the recombination and electron diffusion coefficients, respectively. The drift velocity, $W$ is equal to the production of electric field and charge mobility. $S_{\mathrm{sec}}$ indicates the source term about secondary process, such as photoionization and cathode secondary emission.

With the help of fluid equations, as well as the Poisson equation, the temporal evolution of charge and electric field distribution within the cavity during the discharge development can be obtained. In terms of simulation results, several stages are identified in the discharge development [9, 14, 76], as shown in Figure 6. At first, free electrons are placed in the vicinity of the cathode. Under the action of applied field, they move towards the anode, gaining energy and producing new electrons by impact ionization with neutral gas molecules. At the initial period, the concentration of space charges are not so high to distort the applied field, as Figure 6c. Then the electronic cloud is formed at the avalanche head, as Figure 6d, which spreads out in radial direction due to diffusion and electrostatic repulsion. At this time, the peak number density of charges grows to $10^{19} \mathrm{~m}^{-3}$, and the field distortion is noticeable. Because of a slower drift velocity, the positively charged tail created by positive ions is still located in front of the lower surface of cavity and expands towards the upper surface after the avalanche head arrives at the interface. The electrons in the head land on the lower surface, while secondary electrons generated by photoionization produce new positive ions on the way to the anode, leading to the increase of positive ions within the cavity interior. With the accumulation of positive space charges, a cathode-directed streamer is formed, as Figure $6 \mathrm{~g}$. The streamer propagates to the upper surface, and its arrival leads to the increase of positive surface charges, but the decrease of volume charges, as Figure 6k. At the upper surface, the deployment of positive charges continues at a slow rate due to low values of electric field and consequent drift velocity.
After hundreds of nanoseconds, the deployment of positive surface charges is terminated, and then the charge recombination dominates in the discharge until it stops.

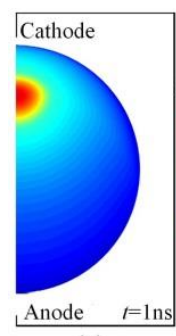

(a)

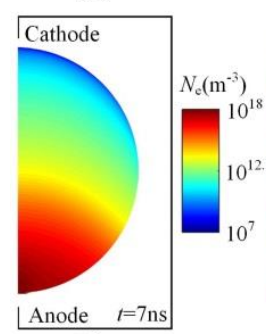

(d)

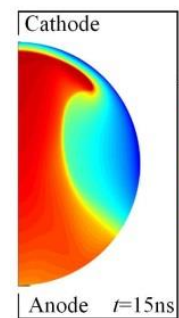

(g)

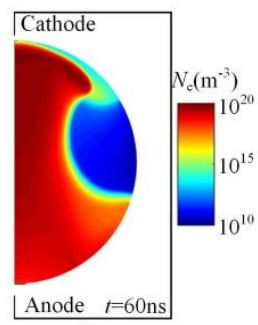

(j)

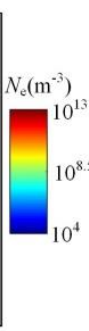

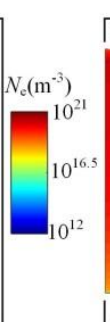
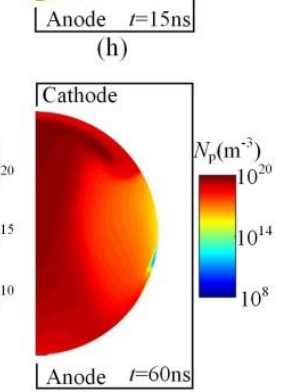

(k)

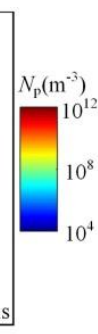

(b)

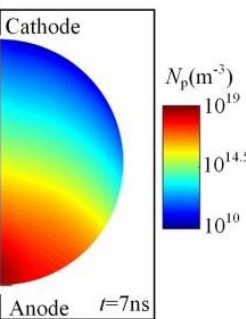

(e)

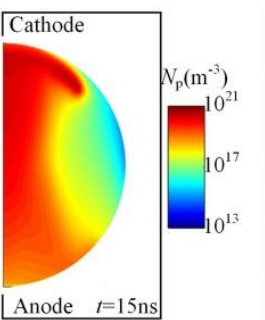

(i)

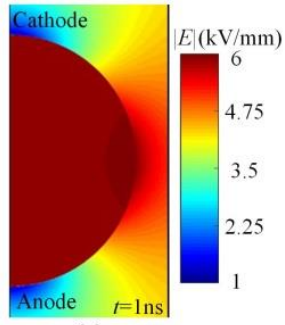

(c)

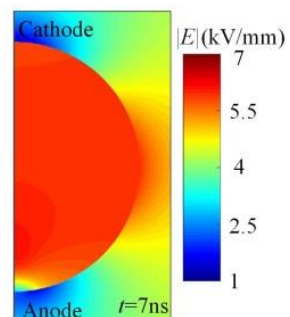

(f)

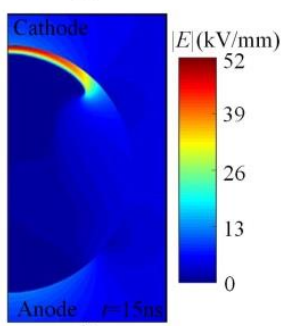

(i)

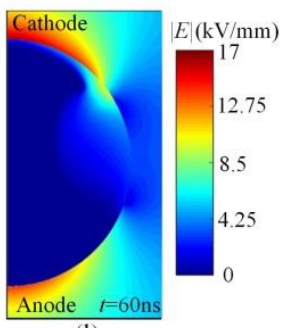

(1)
Figure 6. Time evolution of gas discharge taking place in a spherical cavity with diameter of $1 \mathrm{~mm}$, full of atmospheric pressure air. The potential difference between anode and cathode is $9 \mathrm{kV}$. The first, second and third columns represent the electron concentration, positive ion concentration and electric field distribution, respectively. The first, second, third and fourth rows correspond to the time of $1 \mathrm{~ns}, 7 \mathrm{~ns}, 15 \mathrm{~ns}$ and $60 \mathrm{~ns}$ instantaneously after voltage application, respectively. The figure is reproduced from [14].

As seen from the above physical processes, the photoionization is crucial to the formation of streamer. The photoionization source term $S_{\mathrm{ph}}$ at the position $\boldsymbol{r}$ and time $t$ depends on the photon distribution function $\Psi_{v}(\boldsymbol{r}, \boldsymbol{\Omega}, t)$ of frequency $v$ at position $\boldsymbol{r}$ in the direction $\Omega$ and at time $t$ [77],

$S_{\mathrm{ph}}(\boldsymbol{r}, t)=c \int_{0}^{\infty} \mathrm{d} v \xi_{v}^{\mathrm{ph}} \mu_{\mathrm{abs}, v} \int_{\Omega} \mathrm{d} \Omega \Psi_{v}(\boldsymbol{r}, \boldsymbol{\Omega}, t)$

where $c$ indicates the light speed, $\mu_{\mathrm{abs}, v}$ the function of $v$, represents the absorption coefficient, $\xi_{v}^{\mathrm{ph}}$, in the range of 0 to 1 , is the photoionization efficiency which is equal to the ratio of the number of photoelectrons appearing to the total number of absorbed photons of frequency $v$. In a more physical case, Equation (46) is employed to simulate photoionization so that a 
streamer moving towards the cathode is produced [9, 14]. For purposes of simplified computation, a sparse background of $10^{7}$ $\mathrm{cm}^{-3}$ electron-positive ion pairs is placed uniformly throughout the cavity volume to represent photoionization. In this case, an anode directed streamer will appear [23, 78].

As a more precise description of plasma dynamics, the Boltzmann transport equation is also used to simulate PD activity [79], as follows:

$\frac{\partial N_{\mathrm{e}}}{\partial t}+\frac{\partial N_{\mathrm{e}}}{\partial r} \cdot \frac{p_{\mathrm{e}}}{m_{\mathrm{e}}}+\frac{\partial N_{\mathrm{e}}}{\partial p_{\mathrm{e}}} \cdot F_{\mathrm{e}}=\left.\frac{\partial N_{\mathrm{e}}}{\partial t}\right|_{\text {coll }}$

where $p_{\mathrm{e}}$ is the electron momentum, equal to $m_{\mathrm{e}} W_{\mathrm{e}}, F_{\mathrm{e}}=e E$, the term on the right side of the equation represents the variations in the electron distribution function due to the scattering events associated with the electron collisions with the gas molecules, and $N_{\mathrm{e}}$ is the function of $r, p_{\mathrm{e}}, t$ and $F_{\mathrm{e}}$. Using the Boltzmann transport equation in combination with the fluid and Poisson equations, spatial distributions of electron, ion and electric field can be calculated during the discharge development, as well as the energy density distribution. In terms of this model, Testa et $a l$ found that the hot electrons with a high energy could arrive at cavity surface, and inferred that the bond breaking mechanism is the predominant cause of polymer degradation [79].

Though the fluid model has been widely used, another method named particle in cell (PIC), which estimates the collective behavior of charges in plasma by a small number of superparticles [80], is also attempted for PD simulation. Ganjovi et al developed a two-dimensional PIC based Monte Carlo Collision model to study the dynamics of a streamer-type discharge in a cavity with the size at the level of $100 \mu \mathrm{m}$ [81]. In addition to the information about build-up of space charge within the gaseous channel, consequent modification of the electric field, ionic and electronic currents, and charge accumulation on the electrodes, the phase-space scatter plots for various particle species can be presented.

Compared with others, plasma models provide us more detailed and physical insight into the discharge development of a single PD. However, the consideration of complicated physical processes will bring about modeling difficulty and large computation consumption, which may be the biggest problem for the simulation of multiple PDs. Considering this point, we simulated PD sequences by simplifying streamer propagation [12, 23]. In detail, the photoionization was neglected, and free electrons with a high concentration were placed to shorten the avalanche period. As a consequence, the computation consumption was largely reduced, but the simplification caused significant differences of pulse current waveform and surface charge distribution in comparison with experimental results. In addition, a refined plasma model for continuous PDs was developed by Callender et al, in which the photoionization was taken into account but the effect of residual charges was neglected [26].

\section{DIFFERENCES OF PD MODELING UNDER AC AND DC VOLTAGES}

As we all know, the stationary distribution of electric field depends on dielectric bulk conductivity under DC voltage. Nevertheless, if a PD takes place, generated charges will force the electric field to redistribute, so the permittivity also needs to be taken into account. In this case, the current continuity equation coupled with the Poisson equation is necessary. As for capacitance models, a resistor parallel to each capacitor is added [82, 83], as has been shown in the last row of Table 2. It is found that discharge frequency is related to the time constant for charging the cavity, which is expressed as:

$\tau_{\mathrm{dc}}=\frac{R_{\mathrm{b}} R_{\mathrm{c}}\left(C_{\mathrm{b}}+C_{\mathrm{c}}\right)}{R_{\mathrm{b}}+R_{\mathrm{c}}}$

The expression can be used to explain the dependence of discharge frequency on temperature [84].

There are no differences of discharge development modeling between two types of voltages, because the duration time of single discharge is usually less than $1 \mu \mathrm{s}$, much shorter than $\mathrm{AC}$ voltage cycle. However, in addition to the dependence of electric field distribution, the surface charge dynamics at DC voltage are distinct. Looks back Figure 3, the recombination of surface charges with opposite polarities, as well as the neutralization through gas ions, is difficult to happen due to a constant voltage polarity. Besides, the surface conduction leads to more uniform distribution of charges, but not the decrease of total surface charges. Therefore, it seems that the surface charge decay through insulator volume becomes the predominant way. He et al held that the recovery of cavity field after a discharge is only attributed to the charge decay through volume conduction, and constructed a PD simulation model under DC voltage [85]. In our previous research, surface charge dynamic distributions produced by DC PDs were measured [12]. Similar to the experimental results under $\mathrm{AC}$ voltage, no lateral migration of surface charges was observed. Provided that the volume conduction is the dominant factor for surface charge dissipation, the decaying time will be in the order of hundreds of seconds according to equation (28). However, it was less than $1 \mathrm{~s}$ in experiments. The difference suggests further studies are necessary for surface charge decaying mechanism within the cavity, which is vital to PD modeling especially under DC voltage.

Regardless of voltage waveform, the PD itself acts as a source of charge injecting into the volume of solid dielectrics, which has been verified by experiments and simulations $[9,86]$. Because of unchanged polarity of DC voltage, the space charge accumulation within dielectrics may be gradually enhanced after multiple PDs, leading to more important effect on electric field distribution within the cavity in comparison with $\mathrm{AC}$ voltage. It would be of great interest to take the interaction between gas discharge and space charges into DC PD modeling, but more experimental studies should be carried out in advance.

\section{EXPERIMENTAL VALIDATIONS}

\subsection{MEASURABLE PD PARAMETERS}

In order to correlate simulations with experiments, some measurable PD parameters should be extracted during the simulation. First of all, the apparent charge is necessary, which constitutes phase-resolved PD (PRPD) pattern with discharge phase. PD current pulse waveform is also needed, because it relates to the streamer propagation and discharge area expansion. In addition, although surface charge distribution is not a direct observation for cavity PD, it plays an important role 
in PD activity and can be measured at present [43, 87]. Hence,

Table 3 summarizes how to calculate these parameters in the derivation of surface charge distribution is also considered.

various PD models.

Table 3. Calculation methods of measurable PD parameters for various simulation models.

\begin{tabular}{|c|c|c|c|}
\hline Model & Apparent charge & Discharge current & Surface charge distribution \\
\hline Capacitance & Equation (31) or (32) $[29,49,88]$ & $\begin{array}{l}\text { Calculating the current passing through the resistor } \\
\text { and capacitor related to the cavity based on the } \\
\text { circuit topology }[37,49,56,57] \text {. }\end{array}$ & ( \\
\hline $\begin{array}{l}\text { Electrostatic } \\
\text { analytical }\end{array}$ & Equation $(37)[5,35,39,40]$ & 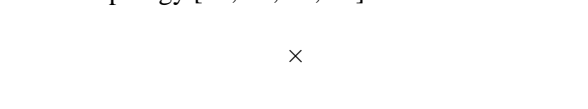 & $\begin{array}{l}\text { A uniform distribution, and the total } \\
\text { surface charges is expressed as equation } \\
\text { (36) [5]. }\end{array}$ \\
\hline $\begin{array}{l}\text { Electrostatic } \\
\text { FEM }\end{array}$ & $\begin{array}{c}q_{\mathrm{app}}=\int_{S_{\mathrm{con}}}\left(\sigma_{\mathrm{con}}^{\mathrm{aft}}-\sigma_{\text {con }}^{\mathrm{bef}}\right) \mathrm{d} S(49)^{\mathrm{a}}[8,60, \\
61]\end{array}$ & $\begin{array}{c}I_{\mathrm{app}}(t)=\frac{\mathrm{d}\left[\int_{S_{\mathrm{con}}} \sigma_{\mathrm{con}}(t) \mathrm{d} S\right]}{\mathrm{d} t}(50) \text { or } \\
I_{\mathrm{app}}(t)=\frac{\mathrm{d} q_{\text {ind }}(t)}{\mathrm{d} t}(51)\end{array}$ & $\begin{array}{l}\text { Charges are added to the cavity surface } \\
\text { until a PD is terminated, so the surface } \\
\text { charge distribution can be directly } \\
\text { obtained [8]. }\end{array}$ \\
\hline Conductance & $\begin{array}{c}q_{\mathrm{app}}=\int_{t_{\mathrm{PD}}}^{t_{\mathrm{PD}}+\Delta t_{\mathrm{PD}}} \mathrm{d} t \int_{S_{\mathrm{con}}} J_{\mathrm{scon}} \mathrm{d} S(52)^{\mathrm{b}}[6, \\
69,70,89]\end{array}$ & $I_{\text {app }}(t)=\int_{S_{\text {con }}} J_{\text {scon }} \mathrm{d} S(53)$ & Equation (27) [41] \\
\hline Plasma & Equation (49) [23] or Equation (52) & $\begin{array}{c}\text { Equation (50) [23], (51) [90], (53) [14] or } \\
I_{\text {app }}(t)=\frac{e}{U_{\text {app }}} \int_{V_{\text {cav }}}\left(N_{\mathrm{p}} \boldsymbol{W}_{\mathrm{p}}-N_{\mathrm{e}} \boldsymbol{W}_{\mathrm{e}}-N_{\mathrm{n}} \boldsymbol{W}_{\mathrm{n}}\right) \mathrm{g} \boldsymbol{E}_{\text {app }} \mathrm{d} V \\
(54)^{\mathrm{c}}[78,90]\end{array}$ & $\begin{array}{c}\sigma_{\mathrm{s}}=\int_{t_{\mathrm{PD}}}^{t_{\mathrm{PD}}+\Delta t_{\mathrm{PD}}} e\left(N_{\mathrm{p}} \boldsymbol{W}_{\mathrm{p}}-N_{\mathrm{e}} \boldsymbol{W}_{\mathrm{e}}-N_{\mathrm{n}} \boldsymbol{W}_{\mathrm{n}}\right) \mathscr{e}_{n} \mathrm{~d} t \\
(55)^{\mathrm{d}}[14,91] \text { or } \\
\sigma_{\mathrm{s}}=e\left(N_{\mathrm{p}}-N_{\mathrm{e}}-N_{\mathrm{n}}\right) \Delta V / \Delta S(56)^{\mathrm{e}}[64]\end{array}$ \\
\hline
\end{tabular}

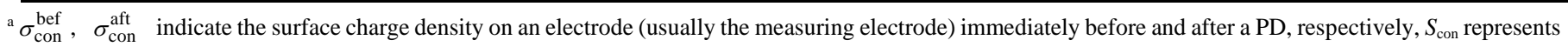
the electrode surface area.

${ }^{\mathrm{b}} t_{\mathrm{PD}}$ is the moment when a PD starts, $\Delta t_{\mathrm{PD}}$ indicates the time duration of PD, $J_{\mathrm{scon}}$ is the current density through an electrode.

${ }^{\mathrm{c}} U_{\text {app }}$ is the applied voltage across electrodes.

${ }^{\mathrm{d}} \boldsymbol{e}_{n}$ indicates the unit normal vector.

e $\triangle V, \triangle S$ are the area and volume of unit grid after meshing, respectively.

The apparent charge is calculated based on the definition of pulsating charges appearing on electrodes when a PD takes place. For a model with the current continuity equation, Equations (49) and (52) are actually identical. The discharge current deduced from circuits is usually characterized as a fast rising edge and a slow decaying tail, similar to the experimental results, but it lacks physical meaning. In Equation (50), the integration of $\sigma_{\mathrm{con}}$ over $S_{\text {con }}$ represents the surface charges on an electrode. Compared with the induced charge in Equation (51), the effect of applied voltage is not eliminated, and it includes additional capacitive component if an AC voltage is applied. Since the PD duration time is very short, Equations (50) and (51) are approximately equivalent, as well as Equation (53). Equation (54) is initially derived in the case of gas discharge across metallic electrodes [92], and then is used in DBD and cavity PD. We compared the pulse current waveform of cavity PD respectively obtained by Equations (51) and (54), and found that they are slightly distinct due to the latter's neglect of surface charge accumulation [90]. Apart from plasma models, the surface charge distribution resulting from simulations is hardly comparable to the experimental results. Equation (55) is recommended, whereas Equation (56) is proposed mainly for the aim of dimensional transformation.

\subsection{REPRODUCTION OF EXPERIMENTAL RESULTS BY SIMULATION}

Due to stochastic behavior of PDs, it is impossible to reproduce experimental results completely by simulations. As a compromise, some statistics, e.g. the average apparent charge,

the discharge frequency and even the shape of PRPD pattern, are taken as evaluation criterion. In general, several parameters are set to be free in simulation models, which are adjusted until a good consistency of the statistics from simulation and experiment is reached. Table 4 summarizes the free parameters and evaluation statistics for simulation under different test conditions. Many parameters are only phenomenological and not directly related to the physical characters of dielectrics, such as $\lambda_{0}, \xi_{+}, \xi_{-}, N_{\mathrm{es} O \mathrm{H}}, N_{\mathrm{es} O \mathrm{~L}}$ and $\delta$, so it is not possible for simulations to give a precise and physical explanation about experimental results even though there is a good consistency of evaluation statistics.

Since studying PD activity in the course of aging is beneficial to the condition monitoring and insulation status assessment of power equipment, it has attracted much attention from various researchers. Up to date, there is a consensus that the evolution of PD behavior with aging is due to the change of gas parameters or cavity surface characteristics mainly based on experiments [94]. However, because of the interaction of the two factors, it is difficult to distinguish the dominant one by experimental methods. For example, there are two kinds of speculations about the appearance of "rabbit-like" PRPD pattern: the change of electronegative gas content [95], and the difference in efficiency to emit a free electron from a positively charged surface or a negatively charged [39]. It is possible to identify and confirm the underlying factors by experiments coupled with simulations, but the related work is extremely scarce.

Table 4. Comparison of simulations with experiments under different test conditions.

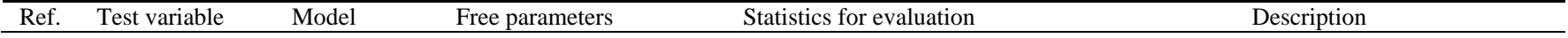


[29]

Aging time Capacitance

$\lambda_{0}^{\mathrm{a}}$

PRPD pattern shape

[39]

Aging time Electrostatic $\quad \Phi, p, \tau_{\mathrm{dt}}, \xi_{+} / \xi_{-}^{\mathrm{b}}$

PRPD pattern shape, $q_{\text {app }}^{\min }, q_{\text {app }}^{\max }$,

$$
N_{\mathrm{HW}}, \triangle \varphi_{0}{ }^{\mathrm{c}}
$$

$S_{\mathrm{k}}(+), S_{\mathrm{k}}(-), K_{\mathrm{u}}(+)$ and $K_{\mathrm{u}}(-)$ for $\mathrm{H}(n$, $q), \mathrm{H}\left(\varphi, q_{\mathrm{m}}\right)$ and $\mathrm{H}(\varphi, n)$ patterns, $I_{\mathrm{PD}}, \Delta \varphi^{\circ}(+), \Delta \varphi^{\circ}(-)$, discharge number acquired in $30 \mathrm{~s}^{\mathrm{d}}$.

Temperature

Electrostatic

$\Phi, \tau_{\mathrm{dt}}$

Voltage

Electrostatic

$\Phi_{0}, \tau_{\mathrm{dt}}, \tau_{\text {work }}$

PRPD pattern shape

[6]

Voltage
frequency

Conductance

$N_{\mathrm{e} 0}, \gamma_{\text {shigh }}, \gamma_{\text {slow }}, U_{\text {ext }}^{\mathrm{e}}$

Discharge number per cycle $\left(N_{\mathrm{AW}}\right)$, PRPD pattern shape

Voltage

amplitude

and cavity

Electrostatic $\quad \rho_{\mathrm{s} 0}, N_{\mathrm{es} 0 \mathrm{H}}, N_{\mathrm{es} 0 \mathrm{~L}}, N_{\mathrm{ev}}, \gamma_{\mathrm{s}}^{\mathrm{f}}$

size

Discharge frequency, total charge

per cycle, $q_{\mathrm{m}}, q_{\mathrm{app}}^{\max }, q_{\mathrm{app}}^{\min }$, PRPD

pattern shape

[93]

Sample
configuratio
Plasma

$\delta, \tau_{\mathrm{ps}}, \tau_{\mathrm{ns}}{ }^{\mathrm{g}}$ $q_{\mathrm{m}}$, discharge frequency
The change of PRPD pattern with aging time is mainly due to the consumption of electronegative gas $\mathrm{O}_{2}$ and variation of residual voltage, and the former is responsible for the appearance of SPMD.

The change of PRPD with aging time is mainly due to a reduction of the gas pressure and several changes of the emissive characteristics of the cavity surface, i.e. $\Phi, \tau_{\mathrm{dt}}, \xi_{+} / \xi$.

The increase of temperature results in a higher discharge magnitude and smaller discharge frequency due to the increase of $\tau_{\mathrm{dt}}$, which could be proposed as an effect of $\Phi$ increase.

All parameters related to dielectrics keep unchanged, because there is no insulation degradation.

The change of PRPD pattern with test frequency is due to the statistical time lag, the charge transport and transition of discharge mechanism in the cavity surface.

Since the electron generation rate increases, the discharge frequency becomes higher when the applied voltage is increased. Because there may be more free charges accumulated along the larger cavity surface after a discharge, surface charge decay could be more significant than the smaller cavity, resulting in a lower electron generation rate and less PDs per cycle.

The difference of decaying time between positive and negative surfaces leads to the positive discharge frequency different from the negative one when a DC voltage is applied to the cavity with an insulating and a metallic surface.

$\mathrm{a} \lambda_{0}$ represents the ratio of occurrence number of discharge per unit phase angle to the cavity overvoltage.

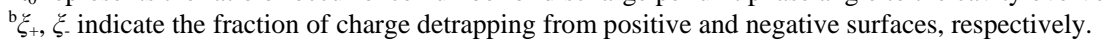

${ }^{\mathrm{c}} N_{\mathrm{HW}}$ represents the average discharge number per ac half cycle, and $\triangle \varphi_{0}$ is the voltage zero overlap.

${ }^{\mathrm{d}} S_{\mathrm{k}}(+)$ and $S_{\mathrm{k}}(-)$ represent the skewness derived from positive and negative discharge patterns, respectively, $K_{\mathrm{u}}(+)$ and $K_{\mathrm{u}}(-)$ are the kurtosis derived from positive and negative discharge patterns, respectively, $I_{\mathrm{PD}}$ is the average discharge current, $\Delta \varphi^{\circ}(+)$ and $\Delta \varphi^{\circ}(-)$ are the voltage discharge phase range for positive and negative discharges, respectively, $\mathrm{H}(n, q)$ indicates the discharge magnitude $v$ s. discharge number pattern, $\mathrm{H}\left(\varphi, q_{\mathrm{m}}\right)$ indicates the mean discharge magnitude $v$. ignition of test voltage, and $\mathrm{H}(\varphi, n)$ indicates the discharge number $v s$. ignition of test voltage.

${ }^{\mathrm{e}} \gamma_{\text {shigh }}$ indicates the surface conductivity when the total amount of charges exceed a critical level, otherwise the surface conductivity is $\gamma_{\text {slow. }}$.

${ }^{\mathrm{f}} \rho_{\mathrm{s} 0}$ indicates the initial surface charge density, $N_{\mathrm{es} O \mathrm{H}}$ is the higher initial electron generation rate due to surface emission, while $N_{\mathrm{es} O \mathrm{~L}}$ is the lower value.

${ }^{\mathrm{g}} \tau_{\mathrm{ps}}, \tau_{\mathrm{ns}}$ are decaying time constants for positive and negative surface charges, respectively.

To the best of the authors' knowledge, the most systematic research was conducted by Gutfleisch et al in 1995 [39], as shown in Figure 7 corresponding to the second row in Table 4. According to the variation of PD characteristics, the aging course from the instant of voltage application to electrical tree initiation is divided into five phases when PRPD patterns are characterized as: A, a horizontal bar; B, a bow-type structure in addition to the horizontal bar (rabbit-like); $\mathrm{C}$, a horizontal bar; $\mathrm{D}$, sinusoidal envelopes; $\mathrm{E}$, similar to the previous phase but with higher discharge repetition rate. For the sake of correctly reproducing the experimental results, several parameters, i.e. $\Phi$, $p, \tau_{\mathrm{dt}}, \xi_{+} / \xi_{-}$, are adjustable. Note that the gas pressure is assumed to play an important role in the PRPD pattern evolution, but the earlier experimental observation [29], as well as the research in recent years [96], suggested that the effect of gas pressure is negligible. On the contrary, the variation of gas composition, e.g. the consumption of $\mathrm{O}_{2}$ and the production of $\mathrm{CO}_{2}$, should be paid attention to. Without considering the change of gas pressure or composition, experimental results are also reproduced by adjusting the parameters related to cavity surface [94]. The divergences indicate that an improved physical simulation model is necessary. In the capacitance model, the free parameter $\lambda_{0}$, far away from the physical essence of PD phenomenon, was used [29], so it is difficult to identify the underling factor.

Another test variable which needs to be highlighted is the voltage frequency due to two reasons. On one hand, in order to reduce power ratings of test equipment, the voltage frequency other than power frequency in PD detection is usually used, e.g. the frequency is reduced when the load is prevailingly capacitive and it is enhanced for the inductive one [97]. In addition, the damped alternating current (DAC) technology has been developed to detect PDs, the frequency of which may range from a few hundred to thousands of $\mathrm{Hz}[98,99]$. The flexibility in the choice of frequency of PD test voltage is based on the study about differences of PD behavior under various voltage frequency. On the other hand, it is significant to clarify memory effect of PD by investigating its activity under different voltage frequency. As the frequency decreases, the time period during which the voltage polarity keeping unchanged will prolong. Therefore, the produced charges by PD will have a longer time to decay, leading to the reduction of memory effect $[94,100,101]$, e.g. the supply of free electrons. 


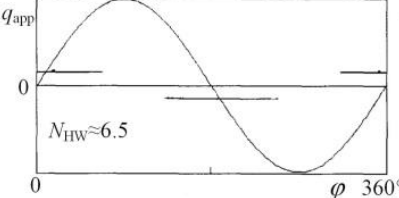

(a) Experiment: phase $\mathrm{A}$

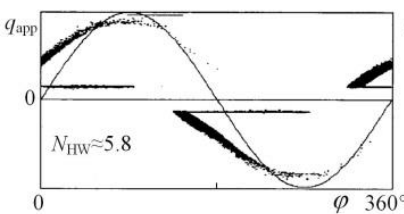

(b) Experiment: phase B

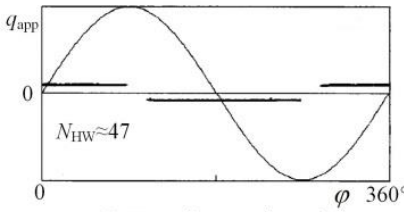

(c) Experiment: phase $\mathrm{C}$

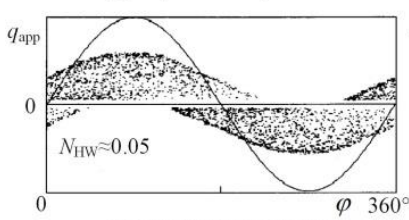

(d) Experiment: phase D

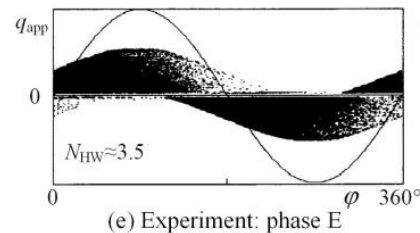

(e) Experiment: phase E

Figure 7. Reproduction of experimental PRPD patterns at different aging phases from simulations. PDs result from a non-vented spherical cavity with the diameter of $2.5 \mathrm{~mm}$ embedded in epoxy resin. At different phases, the free parameters are set as: phase A, $\Phi=1.0 \mathrm{eV}, p=65 \mathrm{kPa}, \tau_{\mathrm{dt}}=2 \mathrm{~ms}, \xi_{+} / \xi_{-}=50$; phase $\mathrm{B}, \Phi=1.1 \mathrm{eV}, p=65 \mathrm{kPa}, \tau_{\mathrm{dt}}=2 \mathrm{~ms}, \xi_{+} / \xi_{-}=50$; phase $\mathrm{C}, \Phi=0.98 \mathrm{eV}, p=6 \mathrm{kPa}, \tau_{\mathrm{dt}}=2$ $\mathrm{ms}, \xi_{+} / \xi_{-}=50$; phase D, $\Phi=1.45 \mathrm{eV}, p=2 \mathrm{kPa}, \tau_{\mathrm{dt}}=1000 \mathrm{~s}, \xi_{+} / \xi_{-}=50$; phase E, $\Phi=1.33 \mathrm{eV}, p=2 \mathrm{kPa}, \tau_{\mathrm{dt}}=1000 \mathrm{~s}, \xi_{+} / \xi_{-}=1$. The figure is reproduced from [39].

A typical result about the comparison of simulation and experimental PRPD patterns at different voltage frequency is presented in Figure 8, corresponding to the fifth row in Table 4, in which the parameters $N_{\mathrm{e} 0}, \gamma_{\text {shigh }}, U_{\text {ext }}$ change with the frequency [6]. When the voltage frequency increases, the values of $N_{\mathrm{e} 0}$ and $\gamma_{\text {shigh }}$ become higher. The tendency suggests that the increase of frequency leads to more supply of free electrons and much faster decay rate of surface charges. The former is in accordance to the enhancement of memory effect. In addition, with the frequency increasing to $100 \mathrm{~Hz}, U_{\text {ext }}$ changes from 10 $\mathrm{V}$ to $2.4 \mathrm{kV}$. The authors attributed the increase to the transition of streamer-like to Townsend-like discharges. This speculation is puzzling and short of direct observation, e.g. the current pulse waveform [102]. In essence, the dielectrics will do not suffer from degradation if the test time is not very long, e.g. less than several hours. Under this case, the parameters relevant to dielectrics should keep unchanged. Based on electrostatic models, A. Cavallini et al. reproduced PRPD patterns at different frequency without adjustment of any parameters [36]. Note that $\tau_{\mathrm{dt}}$ in their model does not only indicate the decay of detrappable charges, but also represents the dissipation of charges which contribute to the electric field.

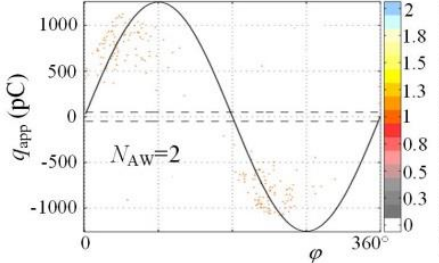

(a) Experiment: $0.01 \mathrm{~Hz}$

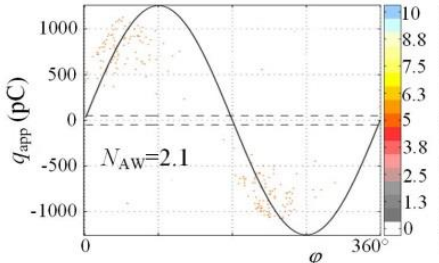

(b) Experiment: $0.1 \mathrm{~Hz}$

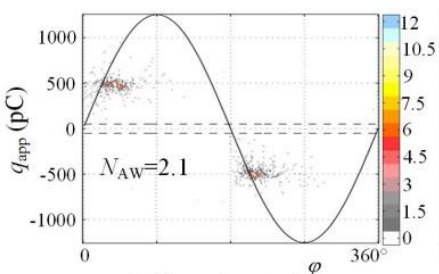

(c) Experiment: $1 \stackrel{\varphi}{\mathrm{H} z}$

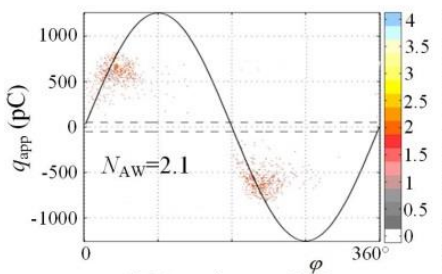

(d) Experiment: $10 \mathrm{~Hz}$

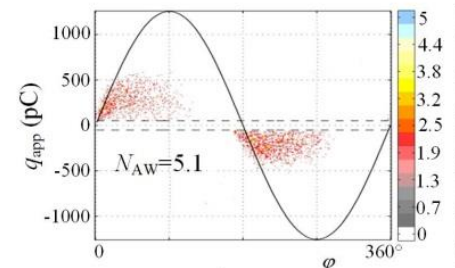

(e) Experiment: $100 \mathrm{~Hz}$ (f) Simulation: $0.01 \mathrm{~Hz}$

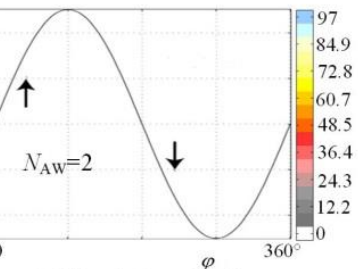

(g) Simulation: $0.1 \mathrm{~Hz}$

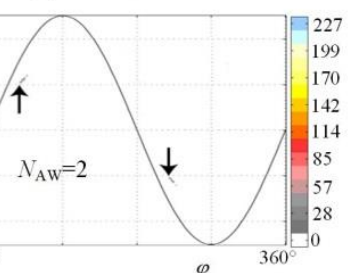

(h) Simulation: $\stackrel{\varphi}{1 \mathrm{~Hz}}$

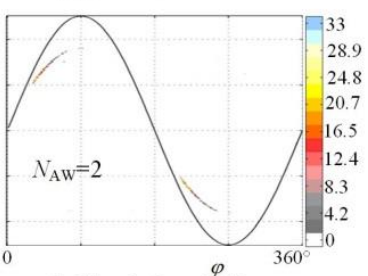

(i) Simulation: $10 \mathrm{~Hz}$

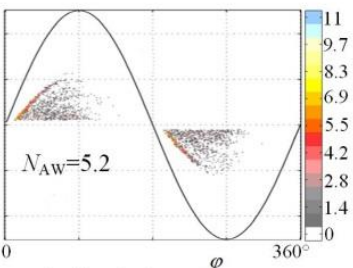

(j) Simulation: $100 \mathrm{~Hz}$

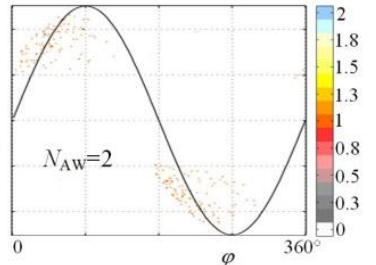

Figure 8. Reproduction of experimental PRPD patterns at different voltage frequency from simulations. PDs result from a cylindrical cavity with the diameter of $10 \mathrm{~mm}$ and the height of $0.5 \mathrm{~mm}$ embedded in epoxy resin. At different frequeny, the free parameters are set as: at $0.01 \mathrm{~Hz}, N_{\mathrm{e} 0}=0.02 \mathrm{~s}^{-1}$, $\gamma_{\text {shigh }}=3 \times 10^{-15} \mathrm{~S}, \gamma_{\text {slow }}=10^{-15} \mathrm{~S}, U_{\text {ext }}=10 \mathrm{~V}$; at $0.1 \mathrm{~Hz}, N_{\mathrm{e} 0}=100 \mathrm{~s}^{-1}, \gamma_{\text {shigh }}=2 \times 10^{-11}$ $\mathrm{S}, \gamma_{\text {slow }}=10^{-15} \mathrm{~S}, U_{\text {ext }}=10 \mathrm{~V}$; at $1 \mathrm{~Hz}, N_{\mathrm{e} 0}=100 \mathrm{~s}^{-1}, \gamma_{\text {shigh }}=2 \times 10^{-11} \mathrm{~S}, \gamma_{\text {slow }}=10^{-15} \mathrm{~S}$, $U_{\text {ext }}=10 \mathrm{~V}$; at $10 \mathrm{~Hz}, N_{\mathrm{e} 0}=100 \mathrm{~s}^{-1}, \gamma_{\text {shigh }}=2 \times 10^{-11} \mathrm{~S}, \gamma_{\text {slow }}=10^{-15} \mathrm{~S}, U_{\text {ext }}=10 \mathrm{~V}$; at $100 \mathrm{~Hz}, N_{\mathrm{e} 0}=500 \mathrm{~s}^{-1}, \gamma_{\text {shigh }}=2 \times 10^{-11} \mathrm{~S}, \gamma_{\text {slow }}=10^{-15} \mathrm{~S}, U_{\text {ext }}=2.4 \mathrm{kV}$. The figure is reproduced from [6].

\section{DISCUSSION}

\subsection{COMBINATION OF DIFFERENT PHYSICAL PROCESSES}

For a complete procedure of PD simulation, several physical processes such as the supply of free electrons, discharge development and surface charge decay should be taken into account. The above four models involve the discharge development process, but not the others, which need the inclusion of these processes. Due to different points of view of the models, the consideration about the combined processes before and after the discharge development may differ from 
each other. Figure 9 shows the procedure of PD simulation, in which the combinations are indicated by the same color. Although the expression of Richardson-Schottky has been applied to capacitance models [37], it is far-fetched to consider free electron supply by volume generation and surface emission, because the both are derived based on the field theory, whereas the capacitance models are on the point of view of circuit.

The process of surface charge decay can be roughly considered by capacitance models, e.g. an exponential function is introduced [54, 103], in which detailed decaying mechanism is not involved. It is difficult to bring the gas ion neutralization into conductance and electrostatic models, because both of them are not relevant to charge transport at all. As for charge decay along the insulator surface, different formulas have been employed, e.g. Equation (26) for the electrostatic analytical model [5] and Equation (57) for the conductance model [6].

$$
\nabla \cdot\left(-\gamma_{\mathrm{s}} \nabla \varphi_{\mathrm{s}}\right)=0
$$

where $\varphi_{\mathrm{s}}$ indicates the electric potential on cavity surface. A more physical formula [104, 105] can be cooperated with electrostatic FEM, conductance and plasma models so that more accurate results may be obtained, as follows:

$\nabla \cdot\left(\gamma_{\mathrm{s}} \boldsymbol{E}_{\mathrm{t}}\right)+\frac{\partial \sigma_{\mathrm{s}}}{\partial t}=0$

where $\boldsymbol{E}_{\mathbf{t}}$ is the vector of tangential electric field on the cavity surface.

In the simulation procedure, the inception and extinction conditions of PD are necessary, but there are several exceptions. For capacitance and analytical electrostatic models, the apparent charge is directly calculated in terms of the extinction voltage or field, so the determination of discharge quenching is not necessary. In addition, since the plasma models are selfconsistent, the extinction condition is redundant.

\subsection{PARAMETER SETTING}

For a simulation model, almost all physical processes are quantitatively described by various equations and logical expressions. Apart from variables, a large number of parameters, e.g. the coefficients related to charge transport in fluid equations, need to be assigned to solve the equations. Values of the parameters may be from more physical calculation or measurement, however, which are sometimes obtained based on other types of discharge, so it should be cautious in employing them. For example, G Callender et al. argued that in Equation (1) the experimental data for the ionization parameters is actually determined from air breakdown between metallic electrodes, whereas the discharge region is frequently bounded by a solid dielectric material in PD systems [14].

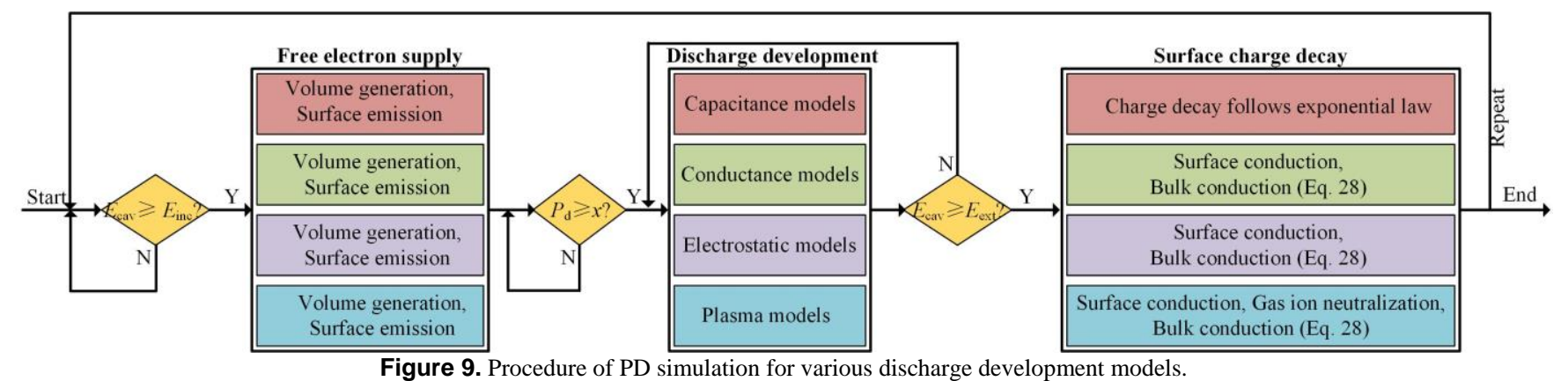

Some parameters, previously mentioned as free parameters, are estimated by comparison of simulation with experiment. On one hand, many of them are only phenomenological and defined according to researchers' own perspective, so it is possible that the definition deviate from physical essence. On the other hand, owing to the usage of multiple free parameters, similar results can be obtained by adjusting different parameters, which may lead to a misunderstanding about some physical phenomena. Therefore, the number of free parameters should be reduced, and it is best to avoid phenomenological parameters.

\subsection{STOCHASTIC NATURE}

PD phenomena are inherently complex stochastic processes that exhibit significant variations in discharge magnitude, discharge time and so on, which have been validated by experiments [40, 106]. Several factors may be attributed to cause the stochastic behaviors, such as the probabilistic nature of the processes that lead to discharge initiation, growth and surface charging dynamics [107]. Except few models based on stochastic process [108, 109], the discharge growth is usually considered to be deterministic in simulations, and the stochastic characteristics are controlled by the two other factors.

In most cases, PD stochastic behavior is simulated by the randomness of free electron supply, as follows. Based on the free electron generation mechanism, the discharge probability $P_{\mathrm{d}}$ is calculated, as shown in Table 1 . Then random numbers generated by computer will be compared with $P_{\mathrm{d}}$ until one of them exceeds it. Due to the introduction of random numbers, the waiting time for free electrons is stochastic, which will lead to the fluctuation of discharge magnitude and time. Since the waiting time is actually discharge time lag, a few researchers also employed it to achieve the stochastic behavior, for example [19]:

$\tau_{\text {lag }}=\left\{\begin{array}{cc}\infty & E_{\text {cav }}<E_{\text {inc }} \\ -\ln \left(1-P_{\mathrm{d}}\right) / \delta & E_{\text {cav }} \geq E_{\text {inc }}\end{array}\right.$

where $\delta$ is the rate parameter of exponential distribution. After the random number $P_{\mathrm{d}}$ is produced, the discharge time lag can be calculated.

As the discharge channel is within $100 \mu \mathrm{m}$, the effect of nonuniform distribution of residual charges and the resultant electrical field have to be taken into consideration in a cavity larger than $100 \mu \mathrm{m}$. Wu et al held that the fluctuation of PD parameters is determined by variation of discharge area of each PD [63]. This viewpoint is inspired by their observation that discharge magnitude has a considerable fluctuation, sometimes 
over 1000 times, which is associated with the charging area but is difficult to explain in terms of free electron supply. They developed a simulation model with the emphasis of discharge area expansion, and found that the surface charged region during a discharge influences the magnitude of subsequent one. It is sufficient to produce variations of discharge magnitude without the inclusion of any stochastic factors.

Table 5. PD initiation locations in different simulation models.

\begin{tabular}{cccc}
\hline Models & Ref. & Initiation location \\
\hline Capacitance & {$[13,37,39,51$,} & {$[5,38]$} & {$[8,60]$} \\
\hline Electrostatic analytical & {$[65]$} & {$[61]$}
\end{tabular}

It should be noticed that PDs always initiate from a point with the maximum electric field in their simulation. This assumption means the PD initiation location would alter during PD sequences, which has been verified by our experimental observation [87]. Moreover, in addition to the stochastic characteristics of free electron generation, the variation of PD location becomes more ambiguous, because PD occurrence may be at any point beyond the inception field where the condition about free electrons is simultaneously satisfied. It is a consequence of PD memory effects mentioned later, and in turn contributes to the stochastic behavior of PD. Anyway, since the statistical characteristics of PDs are important in PD diagnosis, it should be taken into account in simulations so as to pursue the stochastic nature of PDs.

Table 5 summarizes how to set PD initiation location of various simulation models in literature. It is found that most researchers set the initiation location at one or two specific points. Exceptionally, Illias et al considered the variation of discharge initiation location in a rectangular cavity, the surface of which was equally divided into nine parts, and the discharge was modelled to occur within any of the regions [61]. For capacitance models, the cavity is always considered as a whole, so there is no ability to distinguish PD location, as well as the electrostatic analytical model. In terms of discharge area and free electron supply, PD initiation location can be altered for the rest of the models.
Besides of the variation of discharge initiation location, the residual charges and active species in the void space may also contribute to stochastic behaviors of following PDs. Therefore, in order to provide a thorough understanding of the stochastic nature of PDs, a more sophisticated plasma model is required to consider the variation of PD initiation location and the physical processes relevant to PD activity.

\subsection{MEMORY EFFECTS}

The memory effects usually refer to influences of residual charges produced by previous discharge on subsequent one during a PD sequence. In detail, after previous PD takes place, the majority of generated charges will land on the cavity surface, and will experience decaying until the moment when subsequent PD occurs. The residual charges influence its characters, e.g. discharge magnitude, discharge phase and initiation location, by affecting electric field within the cavity and providing additional free electrons. The two effects are successfully considered in PD simulations, e.g. equations (10) and (28), so the influences on PD behavior can be quantitatively determined.

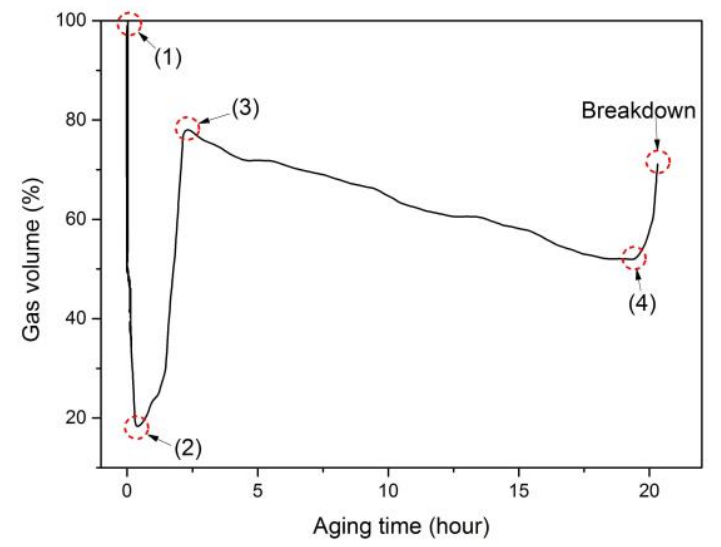

Figure 10. Change of gas volume during the aging process of PD, which takes place in a cylindrical cavity, filled with air, surrounded by LDPE, with a diameter of $3 \mathrm{~mm}$ and height of $02 \mathrm{~mm}$. The figure is reproduced from [95].

Apart from the transient memory effects, permanent changes of discharge site caused by PD aging should also be taken into account, e.g. gas composition, physical and chemical modifications of cavity surface [111], which are named permanent memory effects. The variation of gas composition is mainly attributed to consumption of electronegative gas and production of other gas molecules within the cavity. C. Kim et al. studied the change of gas volume with a constant pressure during PD aging process, which is actually equivalent to the fluctuation of gas pressure with a constant volume [95], as shown in Figure 10. From stage 1 to 2, oxygen molecules are gradually consumed by the oxidation of polymer, leading to the reduction of gas volume. Then the gas volume recovers from stage 2 to 3 due to the production of $\mathrm{CO}_{2}$, which has been verified by gas chromatograph mass spectrometer (GC-MS) measurement [112]. Subsequently, the gas volume slowly decreases since the electronegative gases are consumed. Both the consumption of $\mathrm{O}_{2}$ and production of $\mathrm{CO}_{2}$ would affect the inception time of PD owing to the capture of free electrons by the electronegative gases, but they are rarely taken into account in simulations. 
The cavity surface is modified by PD activity in two ways: chemical reactions and physical attack by bombardment of electrons/ions [1]. One of the modifications is the formation of liquid layer responsible for the increase of surface conductivity. Figure 11 shows the change of epoxy resin surface conductivity [113] and PE surface resistivity [114] with PD aging, which was measured Hudon et al and Morshuis, respectively. It is found that the surface conductivity of epoxy resin increase by at least 7 orders of magnitude during the first 3 hours of exposure, following which tends towards an asymptote in about 800 hours, while the surface resistivity of PE decreases by 6 orders and approximately reaches saturation state in less than $100 \mathrm{~min}$. The increase of surface conductivity will enhance surface charge decay, leading to the change of PD behavior, e.g. the transition of streamer to Townsend mechanism [102]. Although $\gamma_{\mathrm{s}}$ is usually involved in simulation models, its significant change with PD aging measured by experiments has not attracted much attention. It frequently acts a free parameter, so resultant value may largely deviate from the experimental one.

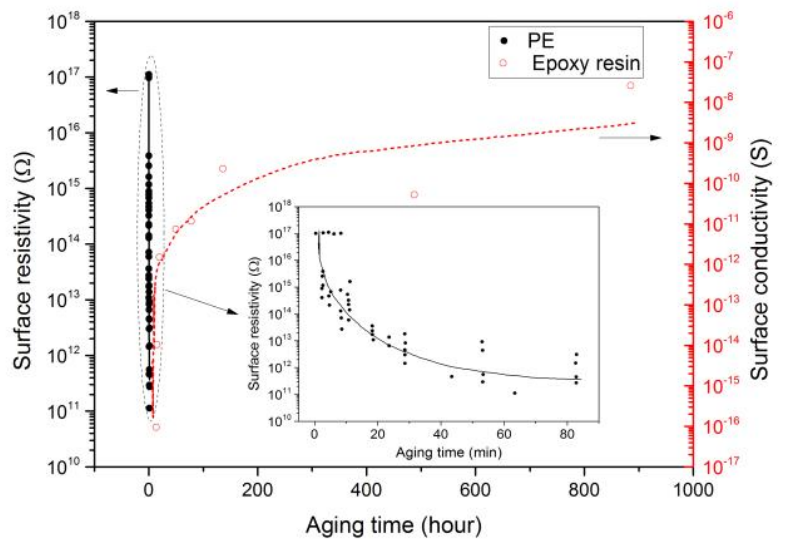

Figure 11. Surface conductivity of epoxy resin and surface resistivity of PE with an exposure to PD. The figure is reproduced from [113, 114].

Other modifications include surface roughness increase and formation of solid by-products. The former is not only related to the latter but also charge carrier bombardment, while the latter results from complicated chemical reactions within the cavity $[1,112,115]$. The increase of surface roughness causes the enhancement of local electric filed, and hence may restrict PD occurrence to some locations, so it is necessary to consider the change in simulations to provide more sight into the aging mechanism. Because of the irregularity of surface morphology (as Figure 12), the introduction to simulation model becomes difficult, but it is possible for a FEM model. Both the physical and chemical modifications, such as surface conductivity and roughness increase, deposition of liquid and solid by-products, may lead to the change of emissive characteristics of charged surface. It is mainly characterized by parameters, such as $\Phi, \tau_{\mathrm{dt}}$ and so on. Because there is short of understanding about the relationship between the parameters and surface status, these parameters are set to be free at the same time in simulation models, possibly arriving at an incorrect conclusion.

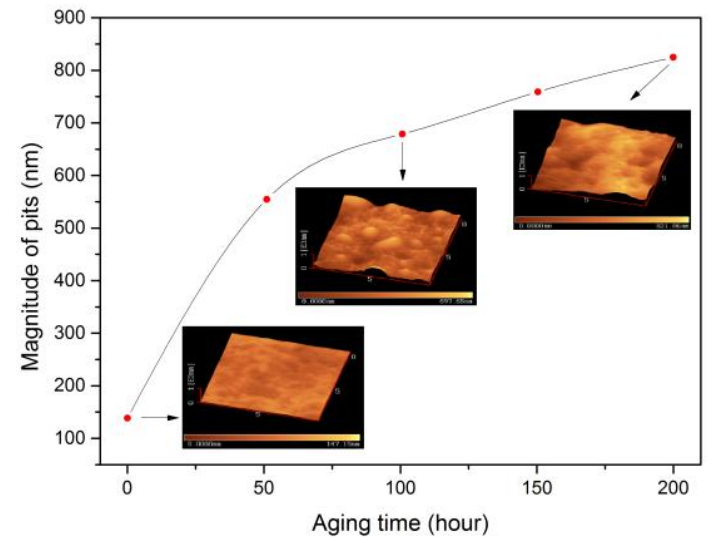

Figure 12. Change of cavity surface pits' maximum magnitude with PD aging. The figure is reproduced from [112].

\section{CONCLUSIONS AND SUGGESTIONS FOR FUTURE WORK}

With the development of thirty years, numerical modeling of cavity PD has been achieved significant progress. Several crucial physical processes to PD phenomena, including free electron supply, discharge development and surface charge decay, are frequently involved in a simulation model. Four models have been put forward to quantitatively describe the discharge development process, i.e. the capacitance, electrostatic, conductance and plasma models, which are based on the points of view of circuit and field, respectively. In terms of the combination of the physical processes and the usage of free parameter, experimental results under various test conditions can be reproduced from simulations. As a result, a better understanding of PD mechanism has been obtained, and the underlying factors which contribute to PD behavior have been identified under different test conditions. However, there are a number of topics that are still not well explored and need further development as follows:

(1) More experimental studies about physical parameters relevant to cavity PD phenomenon are needed to reduce the number of free parameters and avoid incorrect usage of some assigned values which had been deduced from other discharge system. In addition, the understanding about free electron supply, inception and extinction fields need to be further confirmed.

(2) Since it is non-accessible to the cavity, there was no experimental observation about surface charge decay. In recent years, the advent of measurement method based on Pockels effect provides a tool to study it experimentally, but relevant investigations are insufficient. It is urgent to clarify surface charge decaying mechanism in the cavity so that more precise PD simulation model can be constructed, especially for DC PD.

(3) The plasma models are recommended to obtain more physical understanding of PD. Considering the permanent memory effect of PD, existent models should be developed to incorporate physical and chemical changes within the cavity. Further works on PD sequences are still expected to describe the statistical nature of PDs.

(4) With the development of HVDC transmission technique, HVDC power equipment has been widely used. However, it is 
very short of PD numerical modeling under DC voltage. Due to the differences of modeling method under AC and DC voltages, further studies are necessary.

\section{ACKNOWLEDGMENT}

The authors appreciate the financial supports from National Natural Science Foundation of China (51607128), and thank Dr. Yu Gao, Dr. G. Callender for their inspiring discussions.

\section{REFERENCES}

[1] P. H. F. Morshuis, "Degradation of solid dielectrics due to internal partial discharge: Some thoughts on progress made and where to go now," IEEE Trans. Dielectr. Electr. Insul., vol. 12, no. 5, pp. 905-913, Oct. 2005.

[2] T. Tanaka, "Internal partial discharge and material degradation," IEEE Trans. Electr. Insul., vol. 21, no. 6, pp. 899-905, Dec. 1986.

[3] G. C. Stone, "Partial discharge diagnostics and electrical equipment insulation condition assessment," IEEE Trans. Dielectr. Electr. Insul., vol. 12, no. 5, pp. 891-904, Oct. 2005.

[4] T. Okamoto and T. Tanaka, "Analysis of partial discharge characteristics by an equivalent circuit with fluctuating discharge time lag," The Transactions of the Institute of Electrical Engineers of Japan.A, vol. 105, no. 5, pp. 269-275, Oct. 1985.

[5] L. Niemeyer, "A generalized-approach to partial discharge modeling," IEEE Trans. Dielectr. Electr. Insul., vol. 2, no. 4, pp. 510-528, Aug. 1995.

[6] C. Forssen and H. Edin, "Partial discharges in a cavity at variable applied frequency part 2: measurements and modeling," IEEE Trans. Dielectr. Electr. Insul., vol. 15, no. 6, pp. 1610-1616, Dec. 2008.

[7] H. Illias, G. Chen, and P. L. Lewin, "Partial discharge behavior within a spherical cavity in a solid dielectric material as a function of frequency and amplitude of the applied voltage," IEEE Trans. Dielectr. Electr. Insul., vol. 18, no. 2, pp. 432-443, Apr. 2011.

[8] H. A. Illias, G. Chen, and P. L. Lewin, "The influence of spherical cavity surface charge distribution on the sequence of partial discharge events," J. Phys. D: Appl. Phys., vol. 44, no. 24, 245202, Jun. 2011.

[9] Y. V. Serdyuk and S. M. Gubanski, "Computer modeling of interaction of gas discharge plasma with solid dielectric barriers," IEEE Trans. Dielectr. Electr. Insul., vol. 12, no. 4, pp. 725-735, Aug. 2005.

[10] V. Nikonov, R. Bartnikas, and M. R. Wertheimer, "The influence of dielectric surface charge distribution upon the partial discharge behavior in short air gaps," IEEE Trans. Plasma Sci., vol. 29, no. 6, pp. 866-874, Dec. 2001.

[11] A. Villa, L. Barbieri, M. Gondola, A. R. Leon-Garzon, and R. Malgesini, “A PDE-based partial discharge simulator," J. Comput. Phys., vol. 345, pp. 687-705, Sep. 2017

[12] C. Pan, K. Wu, Y. Du, J. Tang, X. T. Tao, and Y. Luo, "Simulation of cavity PD sequences at DC voltage by considering surface charge decay," J. Phys. D: Appl. Phys., vol. 50, no. 20, 205202, Jul. 2017.

[13] H. A. Illias, G. Chen, and P. L. Lewin, "Comparison between threecapacitance, analytical-based and finite element analysis partial discharge models in condition monitoring," IEEE Trans. Dielectr. Electr. Insul., vol. 24, no. 1, pp. 99-109, Feb. 2017.

[14] G. Callender, I. O. Golosnoy, P. Rapisarda, and P. L. Lewin, "Critical analysis of partial discharge dynamics in air filled spherical voids," J. Phys. D: Appl. Phys., vol. 51, no. 12, 125601, Feb. 2018.

[15] K. Wu, Y. Suzuoki, T. Mizutani, and H. K. Xie, "A novel physical model for partial discharge in narrow channels," IEEE Trans. Dielectr. Electr. Insul., vol. 6, no. 2, pp. 181-190, Apr. 1999.

[16] Suwarno, Y. Suzuoki, F. Komori, and T. Mizutani, "Partial discharges due to electrical treeing in polymers: Phase-resolved and time-sequence observation and analysis," J. Phys. D: Appl. Phys., vol. 29, no. 11, pp. 2922-2931, Nov. 1996.

[17] J. V. Champion and S. J. Dodd, "Simulation of partial discharges in conducting and non-conducting electrical tree structures," J. Phys. D: Appl. Phys., vol. 34, no. 8, 1235, Aug. 2001.

[18] L. Niemeyer, B. Fruth, and F. Gutfleisch, "Simulation of Partial Discharges in Insulation Systems," in Int. Symp. High-Voltage Eng. (ISH), 1991, pp. 25-28.

[19] C. Pan, J. Tang, W. Song, Y. Luo, D. Wang, and R. Zhuo, "Investigation of cavity PD physical processes at DC voltage by simulation," IEEJ
Trans. Electr. Electron. Eng., vol. 13, no. 10, pp. 1376-1383, Oct. 2018.

[20] A. Pedersen, I. W. McAllister, G. C. Crichton, and S. Vibholm, "Formulation of the streamer breakdown criterion and its application to strongly electronegative gases and gas mixtures," Archiv für Elektrotechnik, vol. 67, no. 6, pp. 395-402, Nov. 1984.

[21] I. W. McAllister and A. Pedersen, "Corona-onset field-strength calculations and the equivalent radius concept," Archiv für Elektrotechnik, vol. 64, no. 1, pp. 43-48, Jan. 1981.

[22] V. Nikonov, "Influence of electrode surface charge accumulation upon partial discharge behavior," PhD dissertation, University of Montreal, Montreal, 1999.

[23] C. Pan, Y. P. Meng, K. Wu, Z. H. Han, K. Qin, and Y. H. Cheng, "Simulation of partial discharge sequences using fluid equations," J. Phys. D: Appl. Phys., vol. 44, no. 25, 255201, Jun. 2011.

[24] J. M. Meek and J. D. Craggs, Electrical Breakdown of Gases. John Wiley-Sons Ltd., 1978.

[25] P. Morshuis, A. Cavallini, G. C. Montanari, F. Puletti, and A. Contin, "The behavior of physical and stochastic parameters from partial discharges in spherical voids," in Int. Conf. Prop. Appl. Dielect. Mater. (ICPADM), 2000, pp. 304-309.

[26] G. Callender, T. Tanmaneeprasert, and L. Lewin, "Simulating partial discharge activity in a cylindrical void using a model of plasma dynamics," J. Phys. D: Appl. Phys., in press, 2018.

[27] S. Adili, L. G. Herrmann, and C. M. Franck, "Investigating the Inception Mechanism of Pulsed X-ray Triggered Partial Discharges by Time Resolved Measurements," IEEE Trans. Electr. Insul., vol. 20, no. 5, pp. 1780-1788, Oct. 2013.

[28] A. Sobota, J. H. M. Kanters, E. M. van Veldhuizen, F. Manders, and M. Haverlag, "Statistical time lags in ac discharges," J. Phys. D: Appl. Phys., vol. 44, no. 13, 135203, Apr. 2011.

[29] M. Hikita, K. Yamada, A. Nakamura, T. Mizutani, A. Oohasi, and M. Ieda, "Measurements of partial discharges by computer and analysis of partial discharge distribution by the Monte-Carlo method," IEEE Trans. Electr. Insul., vol. 25, no. 3, pp. 453-468, Jun. 1990.

[30] U. Gafvert, H. Edin, and C. Forssen, "Modelling of partial discharge spectra measured with variable applied frequency," in Int. Conf. Prop. Appl. Dielect. Mater. (ICPADM), 2003, pp. 839-842.

[31] N. Hozumi, H. Michiue, H. Nagae, Y. Muramoto, and M. Nagao, "Timelag measurement of void discharges for the clarification of the factor for partial discharge pattern," in IEEE Anпи. Rep. Conf. Electr. Insul. Dielect. Phenom. (CEIDP), 2000, pp. 717-720.

[32] H. A. Illias, M. A. Tunio, H. Mokhlis, G. Chen, and A. H. A. Bakar, "Determination of partial discharge time lag in void using physical model approach," IEEE Trans. Dielectr. Electr. Insul., vol. 22, no. 1, pp. 463-471, Feb. 2015.

[33] J. C. Devins, "The physics of partial discharges in solid dielectrics," IEEE Trans. Electr. Insul., vol. 19, no. 5, pp. 475-495, Oct. 1984.

[34] R. Candela, G. F. Scimemi, P. Romano, and E. R. Sanseverino, "Analysis of partial discharge activity at different temperatures through an heuristic algorithm," Аnnu. Rep. Conf. Electr. Insul. Dielect. Phenom. (CEIDP), 1999, pp. 202-205.

[35] R. Schifani, R. Candela, and P. Romano, "On PD mechanisms at high temperature in voids included in an epoxy resin," IEEE Trans. Dielectr. Electr. Insul., vol. 8, no. 4, pp. 589-597, Aug. 2001.

[36] A. Cavallini and G. C. Montanari, "Effect of supply voltage frequency on testing of insulation system," IEEE Trans. Dielectr. Electr. Insul., vol. 13, no. 1, pp. 111-121, Feb. 2006.

[37] F. Haghjoo, E. Khanahmadloo, and S. M. Shahrtash, "Comprehensive 3-capacitors model for partial discharge in power cables," Compel-Int. J. Comp. Math. Electr. Electron. Eng., vol. 31, no. 2, pp. 346-368, Mar. 2012.

[38] T. S. Negm, M. Refaey, and A. A. Hossam-Eldin, "Modeling and simulation of internal Partial Discharges in solid dielectrics under variable applied frequencies," Int. Mid. East Powe. Syst. Conf. (MEPCON), 2016, pp. 639-644.

[39] F. Gutfleisch and L. Niemeyer, "Measurement and simulation of PD in epoxy voids," IEEE Trans. Dielectr. Electr. Insul., vol. 2, no. 5, pp. 729743, Oct. 1995.

[40] B. Fruth and L. Niemeyer, "The importance of statistical characteristics of partial discharge data," IEEE Trans. Electr. Insul., vol. 27, no. 1, pp. 60-69, Feb. 1992

[41] H. A. Illias, "Measurement and simulation of partial discharges within a spherical cavity in a solid dielectric material," $\mathrm{PhD}$ dissertation, University of Southampton, Southampton, 2011. 
[42] J. Kindersberger and C. Lederle, "Surface charge decay on insulators in air and sulfurhexafluorid - Part I: Simulation," IEEE Trans. Dielectr. Electr. Insul., vol. 15, no. 4, pp. 941-948, Aug. 2008.

[43] K. Wu, C. Pan, Y. Meng, and Y. Cheng, "Dynamic behavior of surface charge distribution during partial discharge sequences," IEEE Trans. Dielectr. Electr. Insul., vol. 20, no. 2, pp. 612-619, Apr. 2013.

[44] C. Pan, K. Wu, Y. Meng, Y. Cheng, and J. Tang, "The effect of discharge area variation on stochastic characters of PD magnitude," IEEE Trans. Dielectr. Electr. Insul., vol. 24, no. 1, pp. 217-226, Feb. 2017.

[45] J. Kindersberger and C. Lederle, "Surface charge decay on insulators in air and sulfurhexafluorid - Part II: Measurements," IEEE Trans. Dielectr. Electr. Insul., vol. 15, no. 4, pp. 949-957, Aug. 2008.

[46] S. Kumara, Y. V. Serdyuk, and S. M. Gubanski, "Surface charge decay on polymeric materials under different neutralization modes in air," IEEE Trans. Dielectr. Electr. Insul., vol. 18, no. 5, pp. 1779-1788, Oct. 2011.

[47] Z. Achillides, G. E. Georghiou, and E. Kyriakides, "Partial discharges and associated transients: the induced charge concept versus capacitive modeling," IEEE Trans. Dielectr. Electr. Insul., vol. 18, no. 6, pp. 15071516, Dec. 2008.

[48] S. Whitehead, Dielectric breakdown of solids, Clearendon Press, 1952.

[49] M. Mahdipour, A. Akbari, and P. Werle, "Charge concept in partial discharge in power cables," IEEE Trans. Dielectr. Electr. Insul., vol. 24, no. 2, pp. 817-825, Apr. 2017.

[50] R. Bartnikas, "Partial discharges: their mechanism, detection and measurement," IEEE Trans. Dielectr. Electr. Insul., vol. 9, no. 5, pp. 763808 , Oct. 2002.

[51] I. W. McAllister, "Electric field theory and the fallacy of void capacitance", IEEE Trans. Electr. Insul., vol. 26, no. 3, pp. 458-459, Jun. 1991.

[52] H. Suzuki, K. Aihara, and T. Okamoto, "Complex behaviour of a simple partial-discharge model,” Europhys. Lett., vol. 66, no. 1, pp. 28-34, Apr. 2004.

[53] D. P. Agoris and N. D. Hatziargyriou, "Approach to partial discharge development in closely coupled cavities embedded in solid dielectrics by the lumped capacitance model," IEE Proc.-Sci. Meas. Technol., vol. 140, no. 2, pp. 131-134, Apr. 1993.

[54] R. Patsch and F. Berton, "Pulse sequence analysis - a diagnostic tool based on the physics behind partial discharges," J. Phys. D: Appl. Phys., vol. 35, no. 1, pp. 25-32, Jan. 2002.

[55] S. S. Samat, I. Musirin, and A. S. Kusim, "The effect of supply voltage on partial discharge properties in solid dielectric," in IEEE Int. Pow. Eng. Optim. Conf. (IPEOC), 2012, pp. 490-495.

[56] Y. Z. Arief, W. A. Izzati, and Z. Adzis, "Modeling of partial discharge mechanisms in solid dielectric material," Int. J. Eng. Innovat. Technol., vol. 1, no. 4, pp. 315-320, Apr. 2012.

[57] C. Y. Ren, Y. H. Cheng, P. Yan, Y. H. Sun, and T. Shao, "Simulation of partial discharges in single and double voids using simulink," in Int. Powe. Modula. Symp. (IPMS), 2006, pp. 120-123.

[58] A. Pedersen, G. C. Crichton, and I. W. McAllister, "The functional relation between partial discharges and induced charge," IEEE Trans. Dielectr. Electr. Insul., vol. 2, no. 4, pp. 535-543, Aug. 1995.

[59] A. Pedersen, G. C. Crichton, and I. W. McAllister, "The theory and measurement of partial discharge transients," IEEE Trans. Electr. Insul., vol. 26, no. 3, pp. 487-497, Jun. 1991.

[60] H. A. Illias, M. A. Tunio, A. H. A. Bakar, H. Mokhlis, and G. Chen, "Partial discharge phenomena within an artificial void in cable insulation geometry: experimental validation and simulation," IEEE Trans. Dielectr. Electr. Insul., vol. 23, no. 1, pp. 451-459, Feb. 2016.

[61] H. A. Illias, M. A. Tunio, H. Mokhlis, G. Chen, and A. H. A. Bakar, "Experiment and modeling of void discharges within dielectric insulation material under impulse voltage," IEEE Trans. Dielectr. Electr. Insul., vol. 22, no. 4, pp. 2252-2260, Aug. 2015.

[62] S. J. Dodd, "A deterministic model for the growth of non-conducting electrical tree structures," J. Phys. D: Appl. Phys., vol. 36, no. 2, pp. 129141, Jan. 2003.

[63] K. Wu, Y. Suzuoki, and L. A. Dissado, "The contribution of discharge area variation to partial discharge patterns in disc-voids," J. Phys. D: Appl. Phys., vol. 37, no. 13, pp. 1815-1823, Jul. 2004.

[64] C. Pan, J. Tang, and K. Wu, "The effect of PD process on the accumulation of surface charges," IEEE Trans. Plasma Sci., vol. 44, no. 11, pp. 2545-2552, Nov. 2016.

[65] H. A. Illias, M. A. Tunio, A. H. A. Bakar, H. Mokhlis, and G. Chen, "Partial discharge behaviours within a void-dielectric system under square waveform applied voltage stress," IET Sci. Meas. Technol., vol. 8, no. 2, pp. 81-88, Apr. 2014.

[66] C. Forssen and H. Edin, "Modeling of a discharging cavity in a dielectric material exposed to high electric fields," presented at the FEMLAB Conference, Stockholm, Sweden, 2005.

[67] S. Matsumura and S. L. Chen, "Effect of plasma resistance on electron temperature measurement by means of an electrostatic probe," J. Appl. Phys., vol. 43, no. 8, pp. 3357-3361, Aug. 1972.

[68] A. L. Kupershtokh, D. I. Karpov, D. A. Medvedev, C. P. Stamatelatos, V. P. Charalambakos, and E. C. Pyrgioti, "Stochastic models of partial discharge activity in solid and liquid dielectrics," IET Sci. Meas. Technol., vol. 1, no. 6, pp. 303-311, Dec. 2007.

[69] H. A. Illias, G. Chen, A. H. A. Bakar, H. Mokhlis, and M. A. Tunio, "Partial discharges within two spherical voids in an epoxy resin," J. Phys. D: Appl. Phys., vol. 46, no. 33, 335301, Aug. 2013.

[70] O. E. Gouda, A. A. ElFarskoury, A. R. Elsinnary, and A. A. Farag, "Investigating the effect of cavity size within medium-voltage power cable on partial discharge behaviour," IET Gener. Transm. Distrib., vol. 12, no. 5, pp. 1190-1197, Aug. 2018.

[71] H. A. Illias, G. Chen, and P. L. Lewin, "Partial discharge within a spherical cavity in a dielectric material as a function of cavity size and material temperature," IET Sci. Meas. Technol., vol. 6, no. 4, pp. 52-62, Aug. 2012.

[72] R. Morrow and J. J. Lowke, "Streamer propagation in air," J. Phys. D: Appl. Phys., vol. 30, no. 4, pp. 614-627, Feb. 1997.

[73] D. Braun, V. Gibalov, and G. Pietsch, "Two-dimensional modelling of the dielectric barrier discharge in air," Plasma Sources Sci. Technol., vol. 1, no. 3, pp. 166-174, Aug. 1992.

[74] J. P. Novak and R. Bartnikas, "Effect of dielectric surfaces on the nature of partial discharges," IEEE Trans. Dielectr. Electr. Insul., vol. 7, no. 1, pp. 146-151, Feb. 2000

[75] V. Nikonov, R. Bartnikas, and M. R. Wertheimer, "Surface charge and photoionization effects in short air gaps undergoing discharges at atmospheric pressure,” J. Phys. D: Appl. Phys., vol. 34, no. 19, pp. 29792986, Oct. 2001.

[76] M. Sjoberg, Y. V. Serdyuk, S. M. Gubanski, and M. A. S. Leijon, "Experimental study and numerical modelling of a dielectric barrier discharge in hybrid air-dielectric insulation," J. Electrost., vol. 59, no. 2, pp. 87-113, Sep. 2003.

[77] C. Julien, S. Pierre, B. Anne, C. Sébastien, and P. Sergey, "The finite volume method solution of the radiative transfer equation for photon transport in non-thermal gas discharges: application to the calculation of photoionization in streamer discharges," J. Phys. D: Appl. Phys., vol. 41, no. 23, 234018, Sep. 2008.

[78] J. Li and S. K. Dhali, "Simulation of microdischarges in a dielectricbarrier discharge,” J. Appl. Phys., vol. 82, no. 9, pp. 4205-4210, Nov. 1997.

[79] L. Testa, S. Serra, and G. C. Montanari, "Advanced modeling of electron avalanche process in polymeric dielectric voids: Simulations and experimental validation," J. Appl. Phys., vol. 108, no. 3, 034110, Aug. 2010.

[80] J. P. Verboncoeur, "Particle simulation of plasmas: review and advances," Plasma Phys. Control. Fusion, vol. 47, no. 5A, p. A231-A260, 2005.

[81] A. A. Ganjovi, N. Gupta, and G. R. G. Raju, "A kinetic model of a PD pulse within voids of sub-millimeter dimensions," IEEE Trans. Dielectr. Electr. Insul., vol. 16, no. 6, pp. 1743-1754, Dec. 2009.

[82] U. Fromm, "Interpretation of partial discharges at dc voltages," IEEE Trans. Dielectr. Electr. Insul., vol. 2, no. 5, pp. 761-770, Oct. 1995.

[83] P. H. F. Morshuis and J. J. Smit, "Partial discharges at DC voltage: Their mechanism, detection and analysis," IEEE Trans. Dielectr. Electr. Insul., vol. 12, no. 2, pp. 328-340, Apr. 2005.

[84] E. Ildstad and T. Haave, "Conduction and partial discharge activity in HVDC cable insulation of lapped polypropylene films," IEEE Int. Conf. on Solid Dielectr. (ICSD), 2001, pp. 137-140.

[85] M. He, M. He, G. Chen, X. Chen, W. Li, C. Zhang, H. T. Wang, M. Y. Zhou, and X. Z. Lei, "Numerical modelling on partial discharge in HVDC XLPE cable,” Compel-Int. J. Comp. Math. Electr. Electron. Eng., vol. 37, no. 2, pp. 986-999, Mar. 2018.

[86] S. I. Jeon, S. H. Nam, D. S. Shin, I. H. Park, and M. K. Han, "The correlation between partial discharge characteristics and space charge accumulation under ac voltage," Annu. Rep. Conf. Electr. Insul. Dielect. Phenom.(CEIDP), 2000, pp. 653-656

[87] C. Pan, K. Wu, Y. Du, Y. P. Meng, Y. H. Cheng, and J. Tang, "The effect of surface charge decay on the variation of partial discharge location," 
IEEE Trans. Dielectr. Electr. Insul., vol. 23, no. 4, pp. 2241-2249, Aug. 2016.

[88] A. Sabt and S. Karmakar, "Simulation of Partial Discharge in High Voltage Power Equipment," Int. J. Electr. Eng. Inform., vol. 3, no. 2, pp. 234-247, Jun. 2011.

[89] G. Chen and F. Baharudin, "Partial discharge modelling based on a cylindrical model in solid dielectrics," in Int. Conf. Condit. Monitor. Diagn. (CMD), 2008, pp. 74-78.

[90] C. Pan, K. Wu, Y. Du, and J. Tang, "Comparison of Sato's equation and Pedersen's theory to obtain gas discharge current," IEEE Trans. Dielectr. Electr. Insul., vol. 23, no. 3, pp. 1690-1698, Jun. 2016.

[91] T. N. Tran, I. O. Golosnoy, P. L. Lewin, and G. E. Georghiou, "Numerical modelling of negative discharges in air with experimental validation," J. Phys. D: Appl. Phys., vol. 44, no. 1, 015203, Aug. 2011.

[92] N. Sato, "Discharge current induced by the motion of charged-particles," J. Phys. D: Appl. Phys., vol. 13, no. 1, pp. L3-L6, Sep. 1980.

[93] C. Pan, W. B. Song, J. Tang, Y. Luo, and X. Y. Luo, "The influence of sample configuration on pd frequency at DC voltage," IEEE Int. Conf. High Voltage Eng. Appl. (ICHVE), 2018

[94] C. Chang, "Degradation by partial discharge in cavities under ac electric field," MS thesis, University of Southampton, Southampton, 2015.

[95] C. Kim, T. Kondo, and T. Mizutani, "Change in PD pattern with aging," IEEE Trans. Dielectr. Electr. Insul., vol. 11, no. 1, pp. 13-18, Feb. 2004.

[96] W. Le, A. Cavallini, G. C. Montanari, and L. Testa, "Evolution of PD patterns in polyethylene insulation cavities under AC voltage," IEEE Trans. Dielectr. Electr. Insul., vol. 19, no. 2, pp. 533-42, Apr. 2012.

[97] C. Nyamupangedengu and I. R. Jandrell, "Partial discharge spectral response to variations in the supply voltage frequency," IEEE Trans. Dielectr. Electr. Insul., vol. 19, no. 2, pp. 521-532, Apr. 2012.

[98] F. J. Wester, E. Guilski, and J. J. Smit, "Detection of partial discharges at different ac voltage stresses in power cables," IEEE Electr. Insul. Mag., vol. 23, no. 4, pp. 28-43, Aug. 2007.

[99] R. Bodega, P. H. F. Morshuis, M. Lazzaroni, and F. J. Wester, "PD recurrence in cavities at different energizing methods," IEEE Trans. Instrum. Meas., vol. 53, no. 2, pp. 251-258, Apr. 2004

[100] A. Cavallini, R. Ciani, M. Conti, P. F. H. Morshuis, and G. C. Montanari, "Modeling memory phenomena for partial discharge processes in insulation cavities," Апnи. Rep. Conf. Electr. Insul. Dielect. Phenom.(CEIDP), 2003, pp. 723-727.

[101] P. Wang, G. Wu, Y. Luo, and G. Zhu, "Effect of repetitive square voltage frequency on partial discharge features," Sci. China-Technol. Sci., vol. 56, no. 6, pp. 1313-1321, Jun. 2013

[102] P. H. F. Morshuis and F. H. Kreuger, "Transition from streamer to Townsend mechanisms in dielectric voids," J. Phys. D: Appl. Phys., vol. 23, no. 12, pp. 1562-1568, Dec. 1990.

[103] R. Patsch and M. Hoof, "Physical modeling of partial discharge patterns," in Int. Conf. Cond. Break. Sol. Dielec., 1998, pp. 114-118.

[104] I. W. McAllister, "Electric fields associated with transient surface currents," J. Appl. Phys., vol. 71, no. 7, pp. 3633-3635, Apr. 1992

[105] I. W. McAllister, "Decay of charge deposited on the wall of gaseous void," IEEE Trans. Electr. Insul., vol. 27, no. 6, pp. 1202-1207, Dec. 1992.

[106] T. Okamoto, T. Kato, Y. Yokomizu, Y. Suzuoki, and T. Tanaka, "PD characteristics as a stochastic process and its integral equation under sinusoidal voltage," IEEE Trans. Dielectr. Electr. Insul., vol. 8, no. 1, pp. 82-90, Feb. 2001

[107] R. J. Vanbrunt, "Stochastic properties of partial-discharge phenomena," IEEE Trans. Electr. Insul., vol. 26, no. 5, pp. 902-948, Oct. 1991.

[108] C. Heitz, "A generalized model for partial discharge processes based on a stochastic process approach," J. Phys. D: Appl. Phys., vol. 32, no. 9 , pp. 1012-1023, May. 1999

[109] R. Altenburger, C. Heitz, and J. Timmer, "Analysis of phase-resolved partial discharge patterns of voids based on a stochastic process approach,” J. Phys. D: Appl. Phys., vol. 35, no. 11, pp. 1149-1163, Jun. 2002.

[110] T. Shahsavarian and S. M. Shahrtash, "Modelling of aged cavities for partial discharge in power cable insulation," IET Sci. Meas. Technol., vol. 9, no. 6, pp. 661-670, Dec. 2015.

[111] R. J. Vanbrunt, E. W. Cernyar, and P. Vonglahn, "Importance of unraveling memory propagation effects in interpreting data on partial discharge statistics," IEEE Trans. Electr. Insul., vol. 28, no. 6, pp. 905916, Dec. 1993.

[112] Y. Sekii, "Degradation of low-density polyethylene and cross-linked polyethylene by partial discharge," IEEE Trans. Dielectr. Electr. Insul., vol. 17, no. 1, pp. 116-124, Feb. 2010

[113] C. Hudon, R. Bartnikas, and M. R. Wertheimer, "Spark-to-glow discharge transition due to increased surface conductivity on epoxy-resin specimens," IEEE Trans. Electr. Insul., vol. 28, no. 1, pp. 1-8, Feb. 1993.

[114] P. H. F. Morshuis, "Partial discharge mechanisms: Mechanisms leading to breakdown, analyzed by fast electrical and optical measurements," $\mathrm{PhD}$ dissertation, Delft University, Delft, 1993.

[115] R. J. Vanbrunt, "Physics and chemistry of partial discharge and corona recent advances and future challenges," IEEE Trans. Dielectr. Electr. Insul., vol. 1, no. 5, pp. 761-784, Oct. 1994.

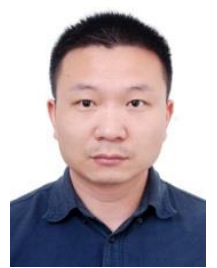

Cheng Pan (S'12-M'17) was born in Guangshui, China, in 1986. He received the B.S. and Ph.D. degrees in electrical engineering from Xi'an Jiaotong University, China, in 2008 and 2014, respectively. Then, he joined the State Grid of China as a Transformer Engineer. After 2015, he became a Lecturer with Wuhan University, China, where he also held a post-doctoral position. From 2017 to 2018, he was with the Tony Davies High Voltage Laboratory, The University of Southampton. His research involves partial discharge mechanism, surface charge accumulation at dc voltage, and breakdown characteristics of moving transformer oil.

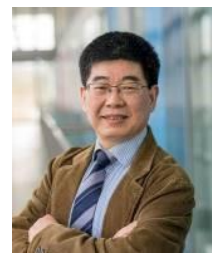

George Chen (SM'11) was born in China in 1961. He received the B.Eng. (1983) and M.Sc. (1986) degrees in electrical engineering from Xi'an Jiaotong University, China. After he obtained the Ph.D. degree (1990) from the University of Strathclyde, UK, on the work of permanent changes in electrical properties of irradiated low-density polyethylene, he joined the University of Southampton as postdoctoral research fellow and became a senior research fellow subsequently. In 1997 he was appointed as a research lecturer and promoted to a Reader in 2002 . He is now the professor of high voltage engineering at the University of Southampton and a visiting professor of Xi'an Jiaotong University. Over the years, he has developed a wide range of interests in high voltage engineering and electrical properties of materials and published over 300 papers. He is active in the HVDC systems and involved with technical working groups in both IEEE and CIGRE.

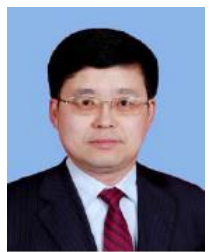

Ju Tang was born in China in 1960. He received the B Eng degree at Xi' an Jiaotong University in 1982, and M Sc. and $\mathrm{Ph}$. D. degrees at Chongqing University in 2000 and 2004, respectively. From 1982, he worked at the School of Electrical Engineering, Chongqing University, and he became a professor in 1998 Currently, he is a professor at the School of Electrical Engineering, Wuhan University. His research interests are on-line monitoring and fault diagnosis technologies about power equipment, gas discharge mechanism and accumulation characters of surface charges.

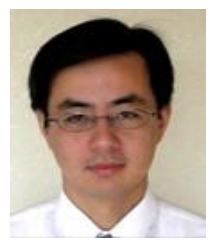

Kai Wu (M'00-SM'13) was born in China in 1969. He received the M.S. and Ph.D. degrees in electrical engineering from Xi'an Jiaotong University, China, in 1992 and 1998, respectively. He was a post-doctoral fellow from 1998 to 2000 and then joined the staff from 2000 to 2003 at Nagoya University, Japan. In 2003, he worked as a research associate at the University of Leicester, UK. In 2004 and 2005, he was a visiting researcher at the Central Research Institute of Electric Power Industry, Japan. Since 2006, he has become a Professor of Xi' an Jiaotong University, China. 